\title{
Experience-Dependent Development of Coordinated Hippocampal Spatial Activity Representing the Similarity of Related Locations
}

\author{
Annabelle C. Singer, ${ }^{1}$ Mattias P. Karlsson, ${ }^{2,3}$ Ana R. Nathe, ${ }^{2}$ Margaret F. Carr, ${ }^{2}$ and Loren M. Frank ${ }^{2}$ \\ ${ }^{1}$ McGovern Institute for Brain Research and MIT Media Lab, Massachusetts Institute of Technology, Cambridge, Massachusetts 02139, ${ }^{2}$ W. M. Keck Center \\ for Integrative Neuroscience and Department of Physiology, University of California, San Francisco, California 94143-0444, and 3Janelia Farms Research \\ Campus, Howard Hughes Medical Institute, Ashburn, Virginia 20147
}

To learn we must identify and remember experiences uniquely but also generalize across experiences to extract common features. Hippocampal place cells can show similar firing patterns across locations, but the functional significance of this repetitive activity and the role of experience and learning in generating it are not understood. We therefore examined rat hippocampal place cell activity in the context of spatial tasks with multiple similar spatial trajectories. We found that, in environments with repeating elements, about half of the recorded place cells showed path-equivalent firing, where individual neurons are active in multiple similar locations. In contrast, place cells from animals performing a similar task in an environment with fewer similar elements were less likely to fire in a pathequivalent manner. Moreover, in the environment with multiple repeating elements, path equivalence developed with experience in the task, and increased path equivalence was associated with increased moment-by-moment correlations between pairs of path-equivalent neurons. As a result, correlated firing among path-equivalent neurons increased with experience. These findings suggest that coordinated hippocampal ensembles can encode generalizations across locations. Thus, path-equivalent ensembles are well suited to encode similarities among repeating elements, providing a framework for associating specific behaviors with multiple locations, while neurons without this repetitive structure maintain a distinct population code.

\section{Introduction}

The world is full of repeating elements, like city blocks or trees evenly spread through a forest. However, we do not understand how neural representations organize these spatial elements. On the one hand, neurons may encode the similarities among these elements to extract general principles about the environment. On the other hand, neurons might encode each element very differently to easily distinguish between them and form unique associations with each element.

We know the hippocampus is required for spatial learning and separating between and generalizing across similar experiences (O'Keefe and Nadel, 1978; Morris et al., 1982; Nakazawa et al., 2002; McHugh et al., 2007). Previous studies of separation and generalization in the hippocampus have examined neural activity as animals navigated similar but distinct environments. In some cases, place cells have different patterns of activity in similar en-

Received Feb. 19, 2010; revised July 4, 2010; accepted July 7, 2010.

This work was supported by the John Merck Fund, the McKnight Foundation, and National Institutes of Health Grants MH077970 and MH080283. We thank Maya Chandru and Patricia Correia for assistance with data collection, and Michael Brainard, Jill Leutgeb, Allison Doupe, Tricia Janak, Sen Cheng, Yuri Dabaghian, Sheri Harris, Shantanu Jadhav, Caleb Kemere, and Steve Kim for their scientific and editorial suggestions.

Correspondence should be addressed to Annabelle C. Singer, McGovern Institute for Brain Research and MIT Media Lab, Massachusetts Institute of Technology, 20 Ames Street, Cambridge,MA 02139. E-mail: asinger@mit.edu.

M. P. Karlsson's present address: Janelia Farms Research Campus, Howard Hughes Medical Institute, Ashburn, VA 20147

DOI:10.1523/JNEUROSCI.0926-10.2010

Copyright $\odot 2010$ the authors $\quad 0270-6474 / 10 / 3011586-19 \$ 15.00 / 0$ vironments (Muller and Kubie, 1987) and can even fire differently in visually identical places (Tanila, 1999). These distinct firing patterns have been termed "global remapping" (Leutgeb et al., 2005b). In other cases, place cells show "rate remapping," where they fire at comparable locations in each environment but at different peak rates (Lever et al., 2002; Leutgeb et al., 2004, 2005b; Wills et al., 2005). Cells can also show a mixture of rate and global remapping (Skaggs and McNaughton, 1998).

Rate remapping appears to be very similar to a pattern of activity termed "path equivalence," where neurons fire in similar locations within and across linear environments. Frank et al. (2000) reported that entorhinal but not CA1 neurons showed path-equivalent activity. In contrast, recent results reported this sort of activity in both the entorhinal cortex (EC) and within the hippocampus (Derdikman et al., 2009). These authors further showed that this activity was associated with a resetting of the grid-cell map at the beginning of each segment of the maze.

Although the presence of these patterns of similar coding is established, the significance of these patterns is unknown. These path-equivalent patterns of activity could be attributable to the similarities in the animal's path through space or behavior (e.g., running direction, turns), or similarities among the geometric elements of the maze. Alternately, the presence of path equivalence or rate remapping in the hippocampus could reflect the animal's inability to distinguish between the environments or paths. Furthermore, it is not clear why these patterns appear in the hippocampus in some cases and not others. We hypothesized 
that these similar coding patterns reflect coordinated, learned generalizations across different places and episodes. We examined neural activity in CA3 and CA1 while animals performed tasks that required distinguishing among similar elements. We found that path equivalence in the hippocampus reflects learned relationships among locations in the animal's environment. Moreover, we found that the firing of pairs of path-equivalent cells was correlated across time, suggesting that they are connected or receiving similar inputs. These findings point to a role for path-equivalent ensembles in encoding and representing learned generalizations.

\section{Materials and Methods}

We combined data from three different studies to examine path equivalence in linear environments. Analyses focusing on different aspects of some of these data were published previously (Karlsson and Frank, 2008, 2009; Singer and Frank, 2009). These studies varied the behavioral task, the geometry of the environment, and the recording location, allowing us to determine which factors influenced place cell activity.

Six-arm maze: pretraining and data collection. Detailed methods are presented in Singer and Frank (2009). Briefly, three male Long-Evans rats were handled and food deprived to $85-90 \%$ of baseline weight. Animals were initially trained to run back and forth on a linear track between food wells where liquid chocolate reward was delivered. Pretraining took place in a different room from the recording room. One of the animals was then pretrained on sequence 1 (S1) in the recording room, whereas two animals were not exposed to the behavioral task until recording began (see Fig. 1a; supplemental Table 1, available at www.jneurosci.org as supplemental material). After pretraining, animals were implanted with a microdrive array containing 16 independently movable tetrodes targeting $\mathrm{CA} 3$ [-3.6 $\mathrm{mm}$ anteroposterior (AP); $3.4 \mathrm{~mm}$ lateral (L)]. Over the next 7-10 d, tetrodes were lowered first to CA1 and then to CA3. CA3 was identified by depth and the characteristic EEG waveforms on each recording tetrode. Tetrodes were then lowered to CA3 until the cell layer was reached. Sharp wave ripples (ripples) and theta oscillations were detected in CA3. For all animals a reference tetrode was positioned in the corpus callosum. All neural signals were recorded relative to that reference to eliminate muscle artifacts from the recordings.

Electrode positions were confirmed by histology (see Fig. 1b; supplemental Fig. 1, available at www.jneurosci.org as supplemental material). For one animal, electrode lesions were made at the end of each tetrode and later confirmed to be in the CA3 pyramidal cell layer. For two animals, the microdrive fell off before lesions could be made. In these animals, we were able to confirm that the implant site was over lateral areas of dorsal CA3 that made it unlikely that tetrodes would pass through the more medial dentate gyrus (supplemental Fig. 1, available at www.jneurosci. org as supplemental material). All tetrode depths were also consistent with CA3 recordings. Furthermore the EEG signatures characteristic of passing through CA1 and then traveling to CA3 were similar in all animals. In particular, as tetrodes traveled to the ventral side of the CA1 layer, the EEG amplitude greatly increased (see Fig. 1c). Thus, although it is possible that a small number of neurons were recorded from the dentate gyrus, we are confident that the large majority of neurons were from CA3. Furthermore, our results were consistent across animals and tetrodes, suggesting that they could not be explained by a small number of neurons recorded in other regions.

Six-arm maze: behavior. These animals learned to perform a sequenceswitching task in a six-arm maze (see Fig. 1a). This task requires that the animal remember and select a correct arm out of a set of geometrically similar, although visually distinct arms. Correct performance in the task is therefore indicative of the animal's ability to distinguish between the arms. Animals were rewarded for correct trajectories with liquid chocolate in reward wells at the end of the arm by an electrically triggered solenoid delivery system. The track included four sequence arms (B, C, $\mathrm{D}$, and $\mathrm{E}$ ) and one extra arm on each end ( $\mathrm{A}$ and $\mathrm{F})$. Arms were separated by vertical walls $(0.6 \mathrm{~cm}$ thick, $24 \mathrm{~cm}$ tall, and $81 \mathrm{~cm}$ long). Distal cues were visible above these walls at either end of each arm and along the straight section connecting different arms. Circles indicate food wells where animals received liquid chocolate reward in arms B through E. Colored arrows indicate trajectories included in sequence 1 (purple) and sequence 2 (orange).

The task consists of two rules. First, a visit to the home $\operatorname{arm}(\operatorname{arm} \mathrm{C}$ in S1 and arm D in S2) was rewarded when the animal came from any other arm (inbound trajectories). Second, a visit to an arm adjacent to the home arm was rewarded when the animal came from the center arm after having previously visited the opposite adjacent arm (outbound alternation). Consecutive repeat visits to the same food well were never rewarded. Together, these rules defined a correct cyclical sequence of food-well visits (see Fig. 1a): right, center, left, center, right, center, left, center, etc. (Frank et al., 2000; Kim and Frank, 2009). If the animal visited an arm not included in the rewarded sequence (e.g., arm A, E, or F for $\mathrm{S} 1$ ), the animal was rewarded after returning to the home arm. During the initial learning of the task, animals learned the inbound component first and then learned to alternate on outbound trajectories. As a result, once animals learned to perform the outbound trajectories with high accuracy, they were generally performing the entire sequence accurately. Rapid learning of the alternation task depends on an intact hippocampus (Kim and Frank, 2009), as does the ability to flexibly alternate among reward contingencies (Hsiao and Isaacson, 1971; Hirsh et al., 1978; Ainge et al., 2007). Thus, hippocampal activity is likely to be important for correct behavior in this task.

During each run session, the animal was placed in the home arm of the to-be-rewarded sequence ( $\operatorname{arm} \mathrm{C}$ for sequence 1 and arm $\mathrm{D}$ for sequence 2 ), but no cues indicated which sequence was rewarded other than the presence or absence of reward at the food wells. Each run session was between 20 and 30 min long; one animal performed two sessions and two animals performed three sessions per day. Rest sessions in a high walled box preceded and followed each run session. Once the animal performed S1 with $80 \%$ accuracy, measured across a run session, or had six full days of training and was above $75 \%$ accurate, the sequence-switching phase of the task commenced. On the first day of sequence-switching, animals first performed one session where S1 was rewarded. Then in the second session, reward contingencies changed such that S2 was rewarded. All subsequent sessions alternated between rewarding S1 and S2 within each day.

We distinguished between "accurate" responses that were consistent with the rules of S1 or S2 and correct (rewarded) responses. This allowed us to score behavior according to the rules of both sequences simultaneously. To illustrate the behavior, we plotted a 20-trial moving average applied to all outbound trials from one animal (see Fig. 1d). We also used a dynamic state-space smoothing algorithm (Smith et al., 2004, 2007) to estimate the animals' probability of an accurate response for each sequence on each trial and to compute confidence intervals for the estimated probability (Singer and Frank, 2009). On the basis of these estimates, we separated each session into two parts: before and after the animals performed the rewarded sequence significantly more accurately than the unrewarded sequence. This point was determined as the trial on which the mode for the probability of correct performance on the rewarded sequence is greater than the confidence bounds for the unrewarded sequence. This allowed us to examine both times when the animals were making many errors and times when their behavior was consistent with the rewarded sequence.

Three-arm maze: pretraining and data collection. The three-arm maze data were recorded from animals 5, 6, and 7 of Karlsson and Frank (2008). Briefly, three male Long-Evans rats (500-600 g) were food deprived to $85-90 \%$ of their baseline weight and trained to run on a linear track with one reward well at each end of the track. Linear track pretraining was performed in a different room from the recording experiments. After the animals were accustomed to behaving for liquid reward (sweetened condensed milk), they were implanted with a microdrive array containing 30 independently movable tetrodes. After 5-6 d of recovery, animals were once again food deprived to $85 \%$ of their baseline weight. The tetrodes were arranged bilaterally in two 15 -tetrode groups centered at $-3.7 \mathrm{~mm} \mathrm{AP}$ and $\pm 3.7 \mathrm{~mm}$ mediolateral. Each group was located inside an oval cannula whose major axis was oriented at a $45^{\circ}$ angle to the midline with the more posterior tip of the oval closer to the midline. 
Tetrodes in the anterior and lateral portions of each group targeted lateral CA3 and more posterior and medial tetrodes targeted CA1.

Three-arm maze: behavior. Each recording day consisted of two or three 15 min run sessions in $\mathrm{W}$-shaped tracks, with rest sessions in a black box before and after each run. The two tracks were geometrically identical but visually distinct and were open to the room but separated from one another by a black barrier (see Fig. $2 a$ ). The tracks had one reward well at the end of each arm, and animals learned to perform the same continuous alternation task described above where, starting from the center arm, they alternated visits to each outer arm for liquid reward (center, left, center, right, and so on) (Frank et al., 2000, 2004; Kim and Frank, 2009). The correct alternation sequence in the three-arm maze was therefore identical to S1 or S2 in the six-arm maze. Errors were not rewarded, and after an incorrect choice of an outer arm, no rewards were given until the animal returned to the center arm. Animals were pretrained on track 1 (T1) for six days and then ran on both $\mathrm{T} 1$ and track 2 (T2) from days 7 onward. Recordings began on day 7. These animals' familiarity with T1 was similar to the animals on the six-arm maze's familiarity with that track, and T2 was initially novel.

Multiple-U maze: pretraining and data collection. Two male LongEvans rats were handled and food deprived to $85-90 \%$ of baseline weight and trained to run on an exposed tabletop for liquid chocolate reward. Pretraining took place in the same room used for recording. After the animals were accustomed to behaving for liquid reward on the exposed tabletop, they were trained on the multiple-U maze (see Fig. $2 b$ ). The arms of each $U$ were separated by a unique divider, and each $U$ was scented uniquely to ensure that the animals could distinguish between them. Based on our experience, the multiple- $U$ task is quite difficult for animals to learn quickly. We therefore introduced the animals to a very short multiple $\mathrm{U}(50 \mathrm{~cm}$ long) (data not shown) and then expanded the environment in two stages. We trained the animals according to a $12 \mathrm{~d}$ protocol, and on each day the animal ran for either two or three $20 \mathrm{~min}$ sessions on the track, and rested in a black box before and after each run session. Days 1-4 of training consisted of two $20 \mathrm{~min}$ runs on the shortest version of the multiple $U$. Days 5-8 consisted of one session on the shortest configuration of the multiple $\mathrm{U}$, a second session on the mediumlength configuration ( $100 \mathrm{~cm}$ long), and a final session on the shortest configuration. On days 9-12, the animals ran the first session on the medium-length configuration, the second session on the longest configuration $(150 \mathrm{~cm}$ long), and the final session on the medium-length configuration (see Fig. $2 b$ ).

After pretraining, animals were implanted with a microdrive array containing 16 independently movable tetrodes targeting CA1 $(-3.6 \mathrm{~mm}$ AP; $2.2 \mathrm{~mm} \mathrm{~L}$ ) using previously described methods (Karlsson and Frank, 2008). Over the next 7-10 d, the tetrodes were lowered to CA1. Seven days after the electrodes were implanted, the animals were run on the open table for liquid chocolate reward until they would eat continuously for two 5 min sessions. We began the experiment after the animals reached this behavioral criterion and the electrodes reached the cell layer. Tetrode positions were adjusted after daily recording sessions for all tetrodes that had poor unit recordings. On rare occasions, some tetrodes were moved before recording sessions, but never within $4 \mathrm{~h}$ of recording.

Multiple-U track: behavior. On each recording day, the animals ran for three 20 min sessions in the medium-long-medium configuration (see Fig. 2b). The first session was the medium-length configuration, the second was the longest configuration, and the third was the mediumlength configuration. All data included for analysis were from the long configuration. The animals were allowed to rest in a black box for $20 \mathrm{~min}$ before and after each session, and we recorded continuously through the rest and behavioral sessions.

Data collection and processing. All data were collected using the NSpike data acquisition system (L. M. Frank, J. MacArthur, Harvard University Instrument Design Laboratory, Cambridge, MA). The animal's position was tracked with an infrared diode array attached to the animal's preamplifier and was reconstructed using a semiautomated analysis of a digital video of the experiment with custom-written software. Spike data were sampled at $30 \mathrm{kHz}$ and digitally filtered between 600 and $6 \mathrm{kHz}$ (two-pole Bessel for high and low pass), and threshold crossing events were saved to disk. Continuous local field potential data from all tetrodes were sampled at $1.5 \mathrm{kHz}$, digitally filtered between 0.5 and $400 \mathrm{~Hz}$, and saved to disk. Ripples were identified as described previously (Cheng and Frank, 2008).

After neural data were collected, individual units were identified by clustering spikes using peak amplitude and spike width as variables. All spike sorting was done using custom software (MatClust; M. Karlsson). It was generally possible to use a single set of cluster bounds defined in amplitude and width space to isolate units across an entire recording session. In the minority of cases where there was a slight shift in amplitudes across time, units (putative single neurons) were clustered only when that shift was coherent across multiple clusters and where plots of amplitude versus time showed a smooth shift. Only well-isolated cells with tightly clustered spikes and clear refractory periods were included. We did not attempt to match cells across days, so in some cases the same cell may have been recorded across multiple days. To help control for the increase in sample size that would result from repeated recordings, we also carried out the main analyses with data from either a single day or data from every other day of recording. All analyses were restricted to putative principal neurons identified using standard criteria (Fox and Ranck, 1981; Frank et al., 2001). The total number of cells recorded was as follows: for the six-arm maze during the switching phase of the task, $n=$ 100,42 , and 128 for animals 1,2 , and 3, respectively; during the initial learning of sequence $1, n=92$ and 160 for animals 2 and 3, respectively; for the three-arm maze, $n=407,607$, and 33 cells for animals 5, 6, and 7, respectively; for the multiple-U track, $n=22$ and 13 cells for animals MU1 and MU2, respectively.

To visualize neural activity across the environments, two-dimensional occupancy-normalized spatial rate maps were constructed with $1 \mathrm{~cm}$ square bins of spike count and occupancy, both smoothed with a twodimensional Gaussian kernel (1.5 cm SD). These maps include times when the animals traveled in both directions in each arm, and were used for visualization only.

For analysis of place field activity, we calculated the "linearized" activity of each cell. Only times when animals were running forward at least 3 $\mathrm{cm} / \mathrm{s}$ were included. The behavioral data were separated into different spatial trajectories (e.g., A to B, B to A, B to C, etc.), and the animal's linear position was measured as the distance in centimeters along the track from the reward site on the start arm. All the trials when the animal was on that trajectory were included to calculate occupancy-normalized firing rate maps. We used $2 \mathrm{~cm}$ spatial bins and smoothed with a $4 \mathrm{~cm}$ standard deviation Gaussian curve with a total extent of $20 \mathrm{~cm}$. Bins with an occupancy of $<0.1 \mathrm{~s}$ were excluded. Cells with a peak linearized firing rate of $>3 \mathrm{~Hz}$ were considered to have a place field on the track and included in additional analyses (cells with place fields, $n=107$ cells in the six-arm maze during the switching phase of the task; $n=108$ cells in the six-arm maze during initial learning of sequence $1 ; n=130$ cells in T1 in the three-arm maze; $n=131$ cells in T2 in the three-arm maze; $n=24$ cells in the multiple- $U$ maze).

We performed a number of analyses to compare single cells' firing in different trajectories. For the three- and six-arm mazes, only correct trials were included. Results were similar if all trials were included. For all mazes, only trajectories in the same turn direction were compared (e.g., right turn trajectories or left turn trajectories) because these are the most similar. This also eliminates the problem of comparing trajectories where the animal crosses the same place in the same direction for one of the arms in the trajectories, as is the case for trajectories that either both start or both terminate in the center arm of the sequence.

To determine whether place fields occurred in similar locations in multiple trajectories, we computed the spatial autocorrelation of each cell's linearized firing. Linearized firing on each trajectory was first normalized by its area so that firing on each trajectory had a total area of one. This allowed us to detect similarities in firing location regardless of absolute rate. Trajectories in the same turn direction were concatenated, and the autocorrelation was calculated. Each correlation was scaled such that the autocorrelation at zero was one.

To determine confidence bounds for these autocorrelations, we computed the cross-correlation between the concatenated normalized linearized firing rate of different cells recorded in the same session. If trajectories had excluded bins in the linearized firing because of low occupancy, they were excluded from the analysis. We computed the 
mean and $90 \%$ confidence bounds for the cross-correlations at each spatial lag. To determine whether cells had peaks in the autocorrelation away from zero, we determined which spatial lags had autocorrelation values above the confidence bounds of the cross-correlations. By definition, a place cell will have a peak in the autocorrelation at lags near zero, so we identified significant lags $>60 \mathrm{~cm}$ or less than $-60 \mathrm{~cm}$ to detect peaks not attributable to autocorrelation near zero lag for each cell. For each cell, two autocorrelations were computed, one for right-turn trajectories and one for left-turn trajectories. Significant lags were identified on both autocorrelations to ensure that periodic cells that had directional firing were properly identified as periodic. Cells with peaks that occurred at lags within $\pm 20 \mathrm{~cm}$ of the length of a trajectory $(140-180 \mathrm{~cm}$ in the six-arm maze and $150-190 \mathrm{~cm}$ in the three-arm maze) in either of the two autocorrelations (left-turn or right-turn trajectories) were considered "periodic" because these cells had firing peaks at distances similar to the length of trajectories in the environment. Cells with significant peaks $>60 \mathrm{~cm}$ but outside of the periodic range were deemed "aperiodic," whereas cells with no significant peak $>60 \mathrm{~cm}$ in both autocorrelations were categorized as "single-peak" cells. When computing the proportion of periodic cells per session, only sessions with at least five isolated cells with place fields on the track were included and all sessions per day were included. When computing the proportion of periodic cells per tetrode, only tetrodes with at least four isolated cells with place fields on the track were included.

To determine the number of significantly path-equivalent trajectory pairs per cell in the six-arm maze, we examined firing on all task-relevant trajectories (trajectories from arm B to C, C to B, C to D, D to C, D to E, and $\mathrm{E}$ to $\mathrm{D}$ ). We compared trajectories in the same turn direction, creating a total of six trajectory pairs. For each pair, the autocorrelations were computed as described above. Pairs with a significant peak in the periodic range were considered path equivalent.

The autocorrelation analysis provides a measure to assign cells to pathequivalent (periodic) or non-path-equivalent (nonperiodic) classes, but it does not provide an obvious way to measure changes in strength of path-equivalent coding over time. We therefore calculated the place field overlap for pairs of cells. Overlap was calculated according to a previously established method (Battaglia et al., 2004). We also calculated normalized overlap where each cell's linearized firing on each trajectory was normalized by its area, and the overlaps between trajectories in the same turn direction were computed. This revealed similarities in firing location in related trajectories regardless of firing rate. Normalized overlap values close to one indicate very similar firing locations, whereas values close to zero indicate very different firing locations. To define cases when firing on two trajectories was in similar locations, we examined trajectories for which the normalized overlap for a pair of linearized firing rates was $>0.3$. We selected 0.3 as a cutoff value because the distributions of normalized overlaps of linearized firing rate from the same cell and different cells cross at this point (see Fig. 3i), but our results were similar for a cutoff value of 0.805 , which was the value representing the beginning of the top $5 \%$ of the same cell distribution.

To examine differences in firing rate, we computed the peak firing rate of the linearized firing of each trajectory. The smaller firing rate was divided by the larger firing rate to create a peak firing rate ratio. Ratios close to one had very similar peak firing rates, whereas ratios close to zero had very different peak firing rates in the compared trajectories. We computed the normalized overlap and peak rate ratio between trajectories in the same turn direction.

We used the normalized overlap measure to examine path equivalence during the initial training on S1 and the first days of exposure to S2 in the six-arm maze. For two animals we recorded neural activity when the animals initially learned S1 from their first exposure to the track until they reached criterion before the sequence-switching phase of the task. During this initial learning period, animals did not know the correct sequence and performed poorly. We therefore included all task-relevant arms and both incorrect and correct trials. We also examined the distributions of normalized overlap for the first days of exposure to T2 in the three-arm maze. In both cases we compared trajectories in the same turn direction.
In addition, we examined the spatial information of place field activity. For each trajectory, we calculated the number of bits per spike according to the formula of Skaggs et al. (1993):

$$
\sum_{i} P_{i}\left(\frac{R_{i}}{R}\right) \log _{2}\left(\frac{R_{i}}{R}\right),
$$

where $i$ indexes over the spatial bins in the trajectory, $P_{i}$ is the probability that the animal was in bin $i, R_{i}$ is the mean firing rate in bin $i$, and $R$ is the mean firing rate over the trajectory. Only trajectories with a peak linearized firing rate of at least $3 \mathrm{~Hz}$ were included to reduce noise caused by low spiking activity. Only the central trajectories that overlapped in both sequences were included to control for potential differences in trajectory sampling between sequences.

We examined moment-by-moment variability in pairs of cells to determine whether there was evidence for sets of path-equivalent cells firing together in organized ensembles. To determine whether neurons' trial-bytrial variability was correlated, we adapted an approach from Schoppik et al. (2008) where we examined the "noise" correlations defined as the correlations of firing rate residuals. The residuals were calculated as the difference, in each $500 \mathrm{~ms}$ window, between the predicted number of spikes from the linearized place field and the actual number of spikes recorded (see below). This approach has a number of advantages over standard cross-correlation techniques. In particular, by examining the correlations of the residuals, we avoid misclassifying increases in overall firing rate caused by place field shape as correlated firing. Instead, measuring the correlation of the residuals of firing asks whether the fluctuations of each neuron about its mean rate are related, as would be the case if the neurons were part of a functional ensemble that receives common inputs.

We divided each recording session into $500 \mathrm{~ms}$ bins, and for each cell we calculated the expected number of spikes in each bin, excluding spikes present during ripples. The expected number of spikes was calculated by first computing the expected firing rate in $33 \mathrm{~ms}$ bins based on the animal's location and the linearized firing curves, and then integrating that rate across each $500 \mathrm{~ms}$ bin. The $500 \mathrm{~ms}$ bin size was chosen to be large enough to effectively average over variability caused by short-time-scale bursting and the modulation of the $\sim 8 \mathrm{~Hz}$ theta rhythm.

We then calculated the residuals: the difference between the expected number of spikes and the actual number of spikes recorded in each time bin. Only bins where the expected firing rate exceeded $0.5 \mathrm{~Hz}$ were included. This cutoff was chosen to avoid floor effects associated with locations where few or no spikes were observed. We computed the correlation between residuals of cell pairs for each trajectory or for the entire session (all trajectories) when animals were running at $>3 \mathrm{~cm} / \mathrm{s}$ and no sharp wave ripples were detected on any tetrode in CA3. Correlations were only computed if there were at least $10 \mathrm{~s}$ of data to correlate, e.g., $\geq 20$ bins in which both cells' expected firing rate was $>0.5 \mathrm{~Hz}$. Residual correlations were compared to the overlap between the cells' linearized firing rates in each trajectory. We also determined whether there was a relationship between the residual correlation for the entire session and the number of trajectories in which two cells overlapped. To compute the number of trajectories in which the cells overlapped, we counted the number of trajectories with an overlap of $>0.3$. In all other trajectories that were not deemed overlapping (e.g., did not have overlap of $>0.3$ ), overlap had to be low, defined as $<0.2$. Cells that had trajectories with overlaps in between these criteria $(0.2-0.3)$ were excluded from this analysis to allow us to classify cell pairs as having either high or low overlap on each trajectory. Finally, to control for possible contamination caused by clustering errors, we repeated all of these residual correlation analyses for cells recorded on different tetrodes. We found similar results with these more restricted data.

We determined the extent to which the hippocampal representation of space preserved information about the uniqueness of each trajectory using population vector correlations (Leutgeb et al., 2005b; Derdikman et al., 2009). Each trajectory was divided into $10 \mathrm{~cm}$ spatial bins, and a population vector of the linearized firing rate for all cells was created. For each animal, recording days were grouped into pairs of consecutive days. Cells from each pair of days in that group were concatenated in the same vector to include more data to compute the correlation. Population vec- 
tors from corresponding spatial bins in different trajectories were correlated. Only trajectories in the same turn direction were compared (e.g., trajectory $\mathrm{B}-\mathrm{C}$ was compared to $\mathrm{C}-\mathrm{D}$ and $\mathrm{C}-\mathrm{B}$ was compared to $\mathrm{D}-\mathrm{C}$ for $\mathrm{S} 1$ ). The resulting correlations from all bins in a pair of trajectories were averaged. We compared these correlations to cases when the cell identity in the population vector was shuffled or the same trajectory was compared across sessions. Only correct trials were included, though results were similar if all trials were included.

Statistics. The measures discussed above tend to produce nonGaussian distributions, so rank-sum tests were used for pairwise statistical tests unless otherwise noted. Similarly, the nonparametric Spearman's correlation was used for all correlation analyses unless otherwise noted. Standard ANOVAs were used when the distribution of the data was not different from a Gaussian distribution (Lillie test, $p>0.05$ ); otherwise, a Kruskal-Wallis one-way nonparametric ANOVA was used.

\section{Results}

We recorded hippocampal neural activity in a six-arm maze (Fig. 1), a three-arm W-shaped maze (Fig. 2a) (Karlsson and Frank, 2008), and a multiple-U track (Fig. 3).

\section{Six-arm maze behavior}

Animals were trained to perform two alternation sequences in a six-arm environment. Rapid learning of the alternation sequence requires the hippocampus (Kim and Frank, 2009); therefore, hippocampal activity is likely involved in performing the six-arm task. Animals first learned a spatial alternation sequence (S1) to criterion and then learned to switch between this sequence and a second alternation sequence (S2) (see Materials and Methods). All six arms remained available for exploration. We initially focused our analysis on the sequence-switching phase of the task, where the entire environment was familiar. When animals switched between sequences, we found that they were using a place-centered strategy as opposed to a body-centered strategy. At the beginning of each session, animals were placed in the center arm of the to-be-rewarded sequence. We found that when animals were first placed in the center arm of S2 they immediately performed the previously rewarded $\mathrm{S} 1$. This can be seen in a quantification of behavior from the first 10 trials in the first session of S2, where animals were more likely to perform S1 than S2 (Fig. 1d,e). Thus, animals used environmental cues and an allocentric reference frame to perform the task, rather than remembering a series of right or left turns based on their body reference frame. These results indicate that the animals were able to distinguish between the different arms of the environment and used this information to perform the task. Thus, when the animal correctly performs the task, similarities in neural coding between arms cannot be explained by a failure to distinguish among similar locations. During this first session on S2, animals executed S1 for several trials and then changed their behavior, eventually learning to perform S2. By the last 10 trials of the session, animals performed S2 with a high degree of accuracy (Fig. 1d,f). Additional quantification of the animals' behavior is presented in Singer and Frank (2009).

\section{Path-equivalent coding in CA3 in the six-arm maze}

The sequence-switching task requires animals to accurately represent their location to receive a reward. We therefore asked whether hippocampal path equivalence was present while animals were learning and performing this task. We examined neural activity in CA3 and restricted our analysis to correct trials. We found that many single cells fired in similar locations across multiple arms in the six-arm environment. We observed pathequivalent activity from single cells in both two-dimensional and linearized firing plots (Fig. $3 a, b$ ). Repetitive firing in different arms and trajectories became more obvious when firing rate maps were scaled so that all firing above $3 \mathrm{~Hz}$ is shown in the same color or, in the case of the linearized firing, when rates were scaled to the peak firing on each trajectory. This scaling emphasizes firing location and disregards peak or total firing.

We found that many cells fired at regular spatial intervals of about the length of a single trajectory. To quantify this activity for each cell ( $n=107$ cells with place fields on the track), we computed an autocorrelation of the cell's linearized firing rate. We normalized the linearized firing rate on each trajectory by its area and then concatenated the linearized firing for trajectories in the same turn direction. The resulting autocorrelations had peaks at zero and for many cells, at about the length of a single trajectory in the environment $(\sim 160 \mathrm{~cm})$ (Fig. $3 c)$. Cells fired at the same spatial separations even though they fired at many different locations in the track. For instance, some cells fired at turns (Fig. $3 a-c$, left), and other cells fired near reward wells (Fig. $3 a-c$, middle), but in both cases these cells fired at $\sim 160 \mathrm{~cm}$ separations.

About half of the cells with place fields on the track had pathequivalent activity as assessed by the autocorrelation measure. To determine that proportion, we compared each cell's autocorrelation to $90 \%$ confidence bounds derived from the distribution of cross-correlations between the linearized firing rates of different cells. Autocorrelations were computed separately for right-turn and left-turn trajectories. We identified the peak of regions where the autocorrelation exceeded the upper 5\% confidence bound and plotted a histogram of peak locations (Fig. $3 d$ ). About half of the cells had significant peaks in either of the two autocorrelograms at lags corresponding to the length of a single trajectory $(140-180 \mathrm{~cm})$, and we categorized these cells as "periodic" (Fig. $3 e$ ). The rest of the cells were either aperiodic, having a significant peak in either autocorrelogram at lags $>60 \mathrm{~cm}$ but not near the length of a single trajectory, or single peak, having no significant peaks in either autocorrelogram at lags $>60 \mathrm{~cm}$. Thus, cells with a single peak for one autocorrelegram but with a significant peak in the other autocorrelegram were classified as either periodic or aperiodic depending on whether the significant peak occurred within the periodic range. These findings indicate that about half of the cells with place fields in the environment showed pathequivalent coding.

This mixture of path-equivalent and non-path-equivalent coding was present within each session on the track and within individual tetrodes, indicating that cells with different firing patterns were present simultaneously (Fig. $3 f, g$ ). Thus, pathequivalent and non-path-equivalent cells are found in the same locations in the hippocampus. This analysis included only correct trials, but path-equivalent activity persisted when we included both correct and incorrect trials (data not shown). To determine the number of trajectories in which a cell's firing was path equivalent, we examined all task-relevant trajectories, both correct and incorrect (see Materials and Methods). During the initial learning of the sequence-switching phase of the task, animals generally explored the six task-relevant trajectories that included all S1 and S2 trajectories, resulting in a total of six trajectory pairs with the same turn direction. Cells most often showed path-equivalent coding in two or fewer trajectory pairs (Fig. 3h), indicating that individual cells were active in a subset of the possible pathequivalent locations. We also found that path equivalence could extend to the outer arms of the six-arm environment (supplemental Fig. 2, available at www.jneurosci.org as supplemental material). 
a
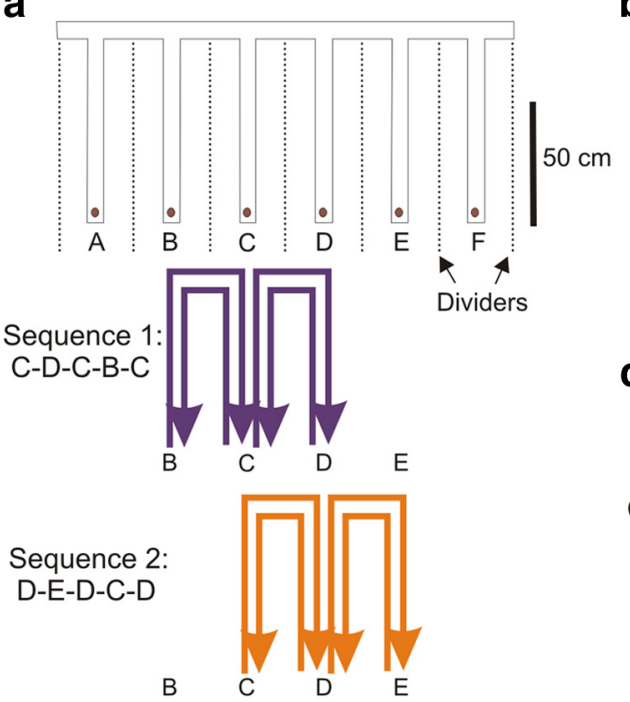

d

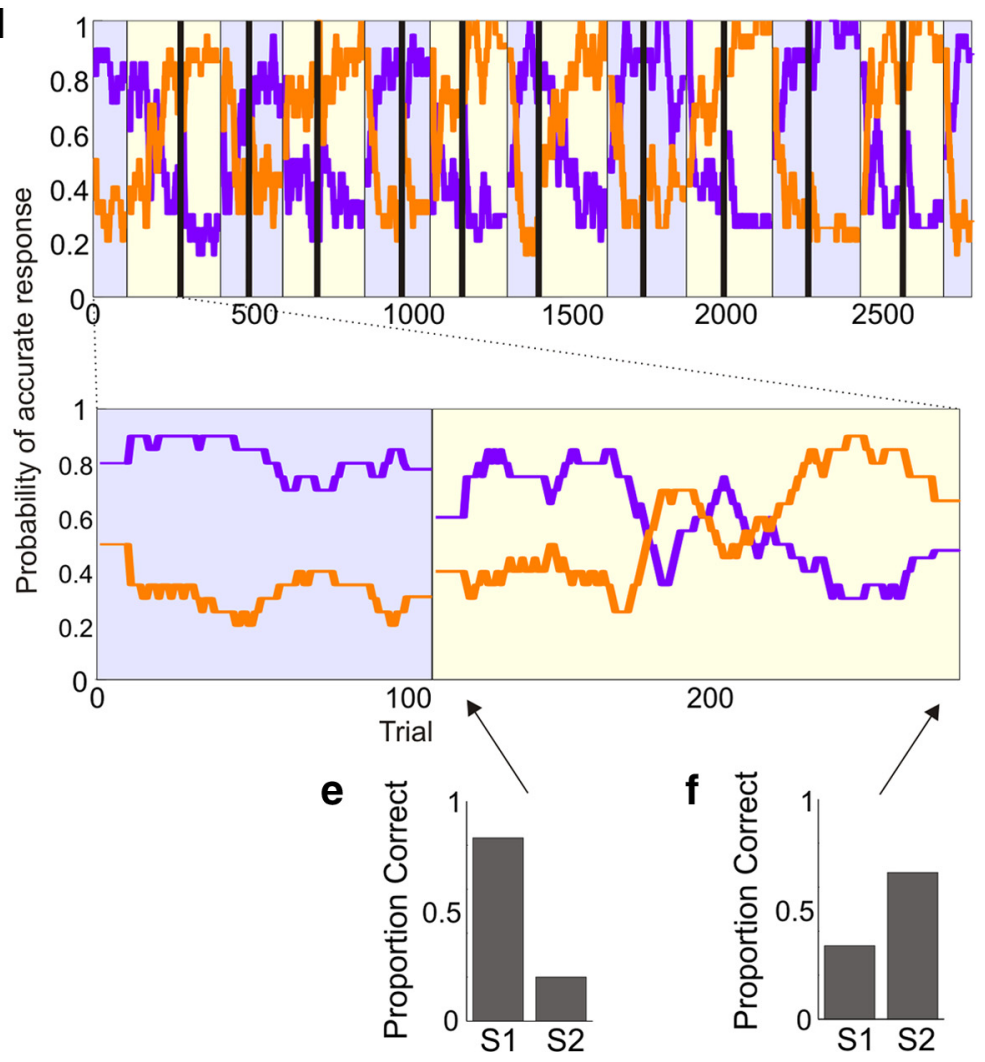

Figure 1. Task design and behavioral performance. $\boldsymbol{a}$, Overhead view of the behavioral apparatus with rewarded sequences indicated by colored arrows (purple, S1; orange, S2). Brown circles indicate the location of food wells; reward was delivered in arms B-E. Dashed lines indicate walls. $\boldsymbol{b}$, Histology showing the hippocampus and tetrode locations. Arrows indicate lesion at the end of a tetrode and the tetrode track. The white outline encompasses the estimated recording area. $c$, All animals showed characteristic EEG signatures (top 3 traces) with large amplitude theta modulation. For comparison, we included a recording from CA1 from the three-arm maze (bottom trace) that is much smaller in amplitude with less prominent theta modulation. Each trace is a total of $1 \mathrm{~s}$ plotted on the same vertical scale. $\boldsymbol{d}$, Twenty-trial moving average of correct responses for one animal when switching between performing S1 (purple) and S2 (orange). Background color indicates which sequence was rewarded. Dark black lines separate recording days. The top graph shows all sequence-switching days, and the bottom graph shows the first day of switching. Chance performance on this task was assumed to be $\sim 0.2$ because there are five arms the animal can choose from when leaving one arm. This value for chance performance is only an approximation because it assumes that the animal was equally likely to sample each arm. , Proportion of the first 10 trials that were correct according to the rules of S1 (left bar) or $\$ 2$ (right bar) in the first session animals were exposed to S2.f, Proportion of the last 10 trials that were correct according to the rules of S1 (left bar) or S2 (right bar) in the first session animals were exposed to $\$ 2$. Trials from all animals were included in $\boldsymbol{e}$ and $\boldsymbol{f}$.

Path equivalence was not attributable to general trends in place field locations (e.g., place fields from many cells congregating around features like turns). We first noted that if path-equivalent activity could be explained by general trends in place field locations, the cross-correlation of concatenated linearized firing from different cells would also show peaks of activity at lags corresponding to the length of a trajectory. This would have broadened the confidence bounds of the distribution, making the threshold for significant autocorrelation peaks higher at the length of a trajectory, and therefore decreased the number cells with this periodic structure.

We also examined this issue by computing the normalized overlap, a measure of the degree of overlap between place field locations, and therefore the degree of path equivalence, as opposed to categorizing the cells as either path equivalent or not. Normalized overlap reflects the similarity of the firing location regardless of firing rate. High normalized overlap occurs when cells fire in very similar locations, whereas low normalized overlap occurs when cells fire in very different locations. We computed the normalized overlap between linearized firing rates for the same cell in two different trajectories and compared that to the normalized overlap of different cells in two different trajectories. We found that the normalized overlap of linearized firing from the same cell between different trajectories was much higher than between different cells on different trajectories using the same set of trajectories in both cases (Fig. 3i) $\left(p<10^{-10} ; n=210\right.$ same-trajectory pairs, 998 different-trajectory pairs; medians, 0.5574 and 0.1661 from the same cell or different cells, respectively). Hence, the presence of path-equivalent firing could not be explained by the clustering of place fields around particular features or more generally by many different cells firing in similar locations in different trajectories.

Furthermore, these analyses also suggest that our results cannot be attributed to poor isolation of single cells. Although poor clustering could produce putative single units that were actually multiple cells, place fields from these cells are not generally present in related locations (Redish et al., 2001), so these multiple fields would not tend to be path equivalent. However, there is one report of anatomical clustering in a very well learned task (Hampson et al., 1999), and it is possible that we would have found clustering if animals had been trained for weeks or 
months on the task. Finally, we noted that because we did not attempt to determine whether we recorded the same cell over multiple days, it is possible that our data include some cells more than once. This is akin to sampling with replacement, and has complex effects on statistical significance. There are no generally accepted methods for determining whether a recorded neuron is the same across days, so we repeated the normalized overlap analysis for single days or every other day of recording to ensure that our results hold for smaller datasets with fewer cells recorded more than once. These analyses produced similar results to those that included all cells across all days: the distribution of normalized overlaps of linearized firing from the same cell on two different trajectories was higher than that of different cells ( $p$ values $<10^{-5}$, ranksum tests).

\section{Path equivalence increases with experience}

The presence of path equivalence during the spatial alternation task in the six-arm maze shows that path equivalence is not attributable to an animal's failure to distinguish between locations, but it is still unclear whether this pattern of firing is likely to serve a functional purpose. If path-equivalent activity reflects the encoding of common behavioral associations across related locations (Frank et al., 2000), we would expect that the strength of path equivalence would increase with experience. Indeed, we found that path equivalence increased with experience relating the paths, both when the environment was novel and when it was more familiar.

We first found that the prevalence of path equivalence increased with experience during the initial training on S1. Because animals did not know the correct sequence and performed poorly, we included both incorrect and correct trials on trajectories in S1, comparing trajectories in the same turn direction. On days 2 through 5, path equivalence for individual neurons, measured as the normalized overlap of linearized firing across different trajectories, increased as animals became more familiar with the environment and learned S1 (Fig. 4a) (KruskalWallis one-way ANOVA, $\chi_{(4,115)}^{2}=17.46, p<0.005$; day 2 was significantly less than days 4 and $5, p<0.05$, Tukey-Kramer post hoc test). Thus, path equivalence increased as animals learned the initial sequence. Animals did not traverse the task arms enough on day 1 to compute linearized firing rates and normalized overlaps.

Similarly, the prevalence of path equivalence also increased when animals learned the new sequence (S2) in the now familiar environment. During learning of S2, the entire environment and all of the task trajectories were familiar, as the animals fully exrewarded sequence.

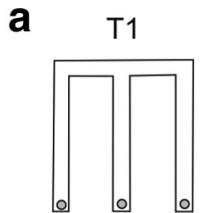

T2

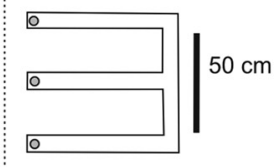

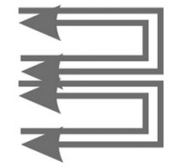

Run 2
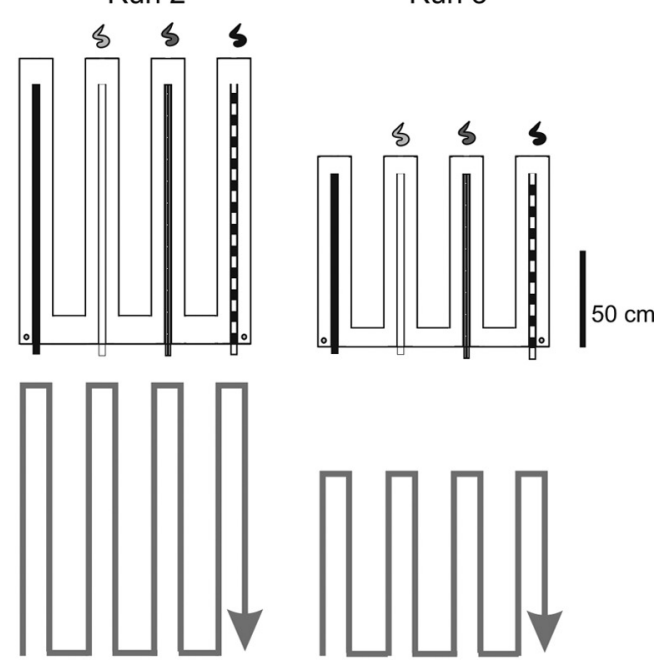

Run 3
Figure 2. Three-arm maze and multiple-U track. $\boldsymbol{a}$, Overhead view of the two three-arm W-tracks. Gray circles denote the location of food wells. Gray arrows indicate trajectories in the rewarded sequence. Animals were rewarded for performing the same

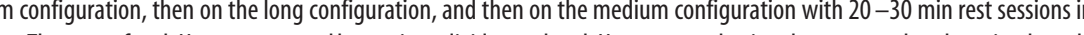
between them. The odors were no scent, bergamot (indicated with light gray symbol above track), cinnamon (dark gray symbol), and lavender (black symbol). Gray circles denote the location of food wells. Gray arrows indicate trajectories in the

plored the environment when initially learning S1. Thus, the CA3 place field map would be expected to be stable (Leutgeb et al., 2004; Karlsson and Frank, 2008). However, some of the task trajectories are specific to S2 and are never rewarded during the initial learning of S1 (trajectories from arm D to E and E to D). As a result, these trajectories do not initially have the same task relevance during the initial learning as trajectories that are included in S1. We examined the normalized overlap between different sets of trajectories during sessions in which S2 was rewarded. Because animals were learning to switch between S1 and S2, they traversed all trajectories in both S1 and S2. We found that the distribution of normalized overlaps for S2 trajectories increased with experience (Figs. $4 b$, left, $c$ ) (Kruskal-Wallis oneway ANOVA; $\left.\chi_{(2,199)}^{2}=13.06 ; p>0.005\right)$. In contrast, the normalized overlaps of pairs of S1 trajectories did not show any 
a<smiles>[111InH]</smiles>

b
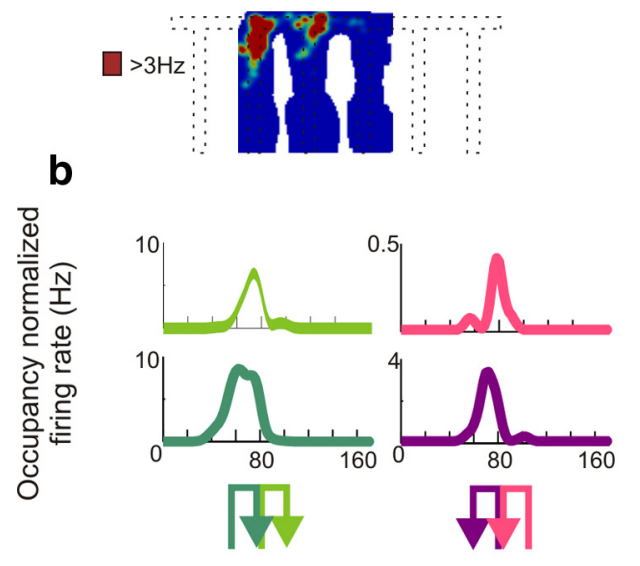

C
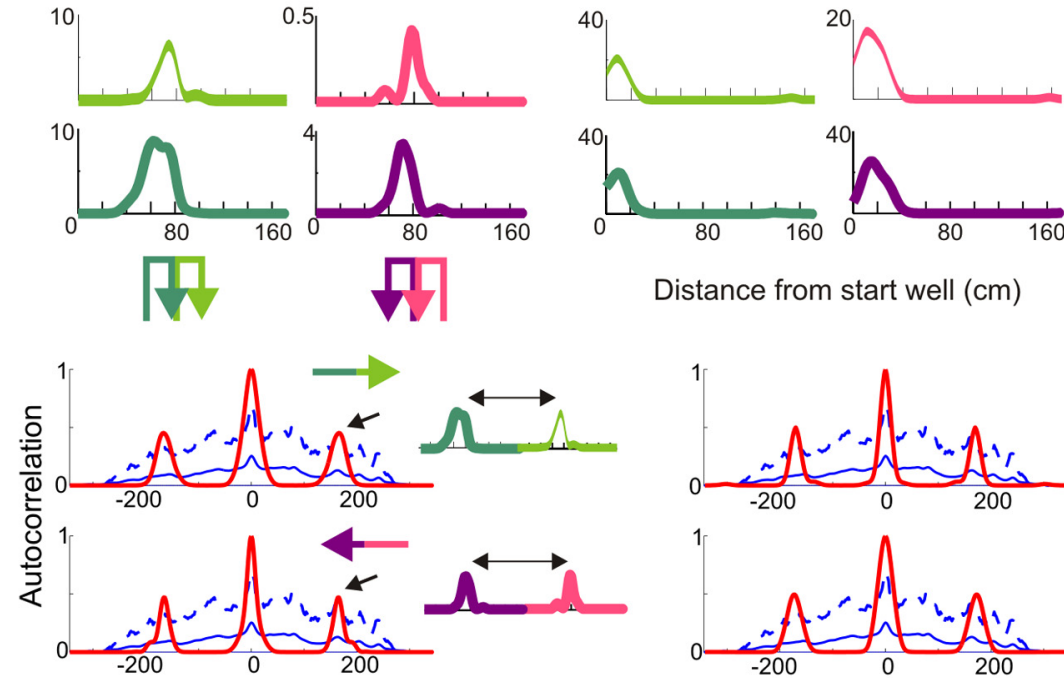

Distance from start well $(\mathrm{cm})$

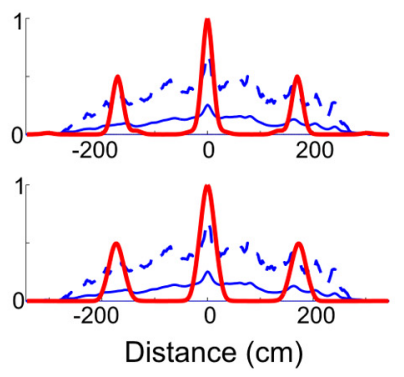

Distance $(\mathrm{cm})$

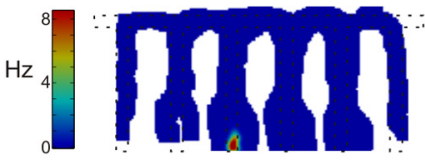

amsm

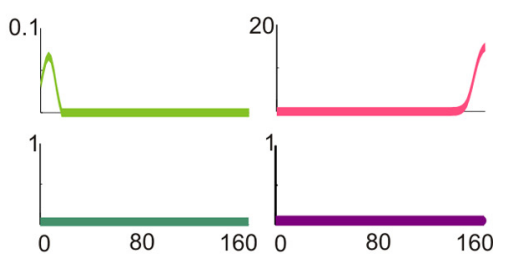

d

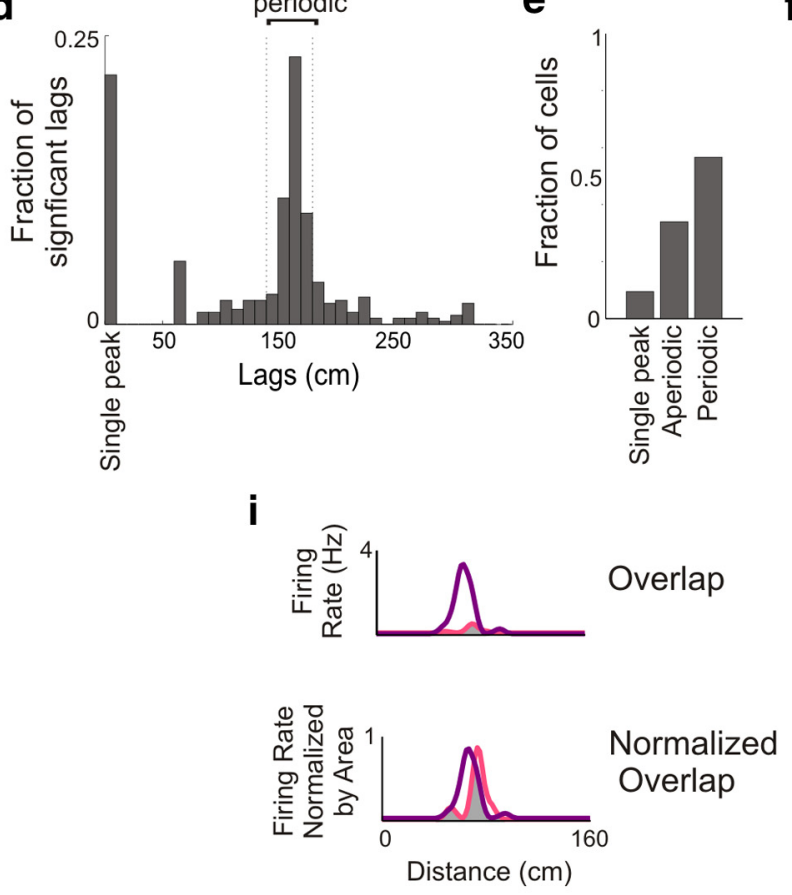

f

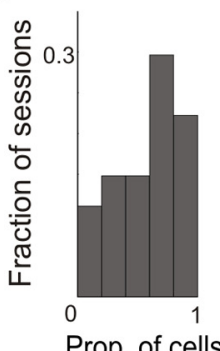

Prop. of cells

per session that were periodic g

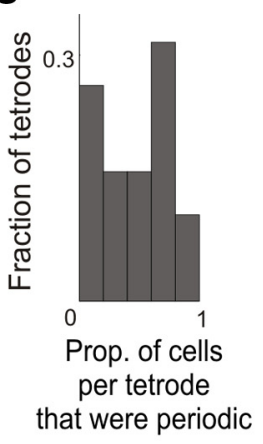

h

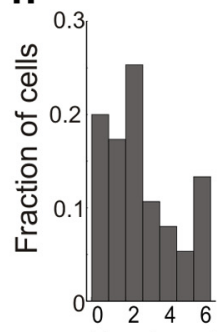

Number of periodic trajectory pairs per cell

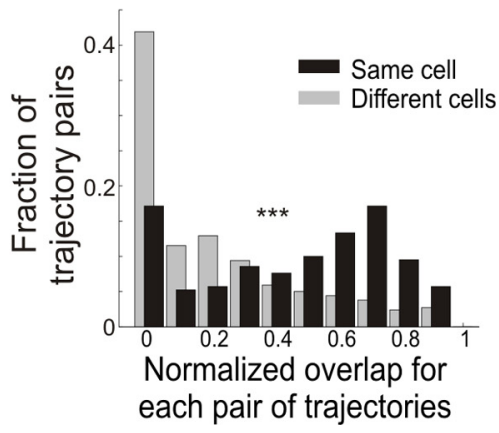

Figure 3. Path-equivalent coding in the six-arm maze. $\boldsymbol{a}$, Spatial firing rate maps of three cells scaled to the cells' peak firing rate (above) or scaled so that all firing $>3 \mathrm{~Hz}$ is red (below). Cells on the left and in the center show path-equivalent firing patterns, whereas the cell shown on the right does not. Only times when the animal was running $>3 \mathrm{~cm} / \mathrm{s}$ are included. $\boldsymbol{b}, 0 \mathrm{ccupancy-}$ normalized firing rates on each linearized trajectory for the same three cells shown in $\boldsymbol{a}$. All trials when the animal traveled on each trajectory are included together to create the linearized firing rates. c. Autocorrelation of the linearized firing for the same three single cells shown in $\boldsymbol{a}$ and $\boldsymbol{b}$ (red lines). Linearized firing rates on trajectories in the same turn direction were normalized by their area and then concatenated (inset). Top graphs show right-turn trajectories as the animal moves from the left to the right (light and dark green arrows and curves), and bottom graphs show left turns as the animal moves from the right to the left (pink and purples arrows and curves). The peak in the autocorrelation (single-headed black arrow) indicates the distance between peaks in the linearized firing concatenated across the two trajectories (double-headed black arrow). To determine whether the peak was significant, the autocorrelation was compared (Figure legend continues.) 
further changes with experience in the sequence-switching phase of the task (Fig. $4 b$, middle) $\left(\chi_{(2,137)}^{2}=3.06 ; p>0.2\right)$. Similarly, comparisons of normalized overlaps between trajectory pairs where one trajectory was unique to $\mathrm{S} 1$ and the other was unique to S2 yielded no significant changes (Fig. $4 b$, right) $\left(\chi_{(2,120)}^{2}=5.28 ; p>\right.$ $0.07)$. Thus, even though animals had many days of experience on the track, when S2 was novel, firing on the pairs of trajectories in S2 was relatively dissimilar. With more experience with S2, the coding for these related, task-relevant trajectories became more similar.

These changes in path equivalence with learning could not be explained by remapping of field location or changes in peak rate associated with the switch from S1 to S2. If remapping occurred, we would expect that neurons would fire differently in the trajectories shared across S1 and S2 when the animal transitioned from performing S1 to performing S2. We therefore measured the normalized overlap from the same cell on the same trajectory when the animal performed S1 versus S2 accurately. We found that the normalized overlap was high, as we would expect if the cells fired in similar locations regardless of the sequence being performed. We compared that distribution to the normalized overlap between different trajectories in the same session. We found significantly higher overlaps across S1 and S2 for the same trajectory than across different trajectories in the same session (Fig. $4 d$ ) $(p<0.001 ; n=118$ same-trajectory pairs in S1 vs S2, $n=210$ different-trajectory pairs in the same session; medians, 0.7123 and 0.5574 from the same trajectories in S1 and S2 or different trajectories, respectively). These results show that firing location on the same trajectory in different sequences was very similar, more similar even than the distribution of firing locations between trajectories that can be path equivalent. Therefore, there was no evidence for changes in place field location between S1 and $\mathrm{S} 2$.

We similarly found no changes in overall peak rate when the animal switched from S1 to S2. We compared peak linearized firing rates on the same trajectory when animals were performing S1 versus S2 and took a ratio of the smaller peak divided by the larger peak firing rate. We compared this rate ratio to that obtained from times when the animal was performing the same sequence across two epochs and found no differences $(p>0.08$; medians, 0.6941 and 0.6072 for S1 and S2, respectively; $n=118$

\section{$\leftarrow$}

(Figure legend continued.) to the distribution of cross-correlations of the linearized firing between different cells (blue; solid line, mean; dashed line, $90 \%$ confidence bounds). $\boldsymbol{d}$, Histogram of significant peaks at nonzero lags for each cell's linearized firing autocorrelations. The region between the dotted lines corresponds to lags that are within $20 \mathrm{~cm}$ of the length of a single trajectory and were therefore classified as periodic. Autocorrelations and significant peaks were computed separately for trajectories in each turn direction. $\boldsymbol{e}$, Proportion of cells that had periodic or aperiodic activity or had no significant peak at nonzero lags (single peak). Periodic cells had significant lags at $150-170 \mathrm{~cm}$ in either autocorrelogram. Single-peak cells had no significant nonzero lags in either autocorrelogram. Aperiodic cells had significant nonzero lags outside of $150-170 \mathrm{~cm}$. $f$, Proportion of cells that had periodic activity in each session, including all sessions per day during the sequence-switching phase of the task. $g$, Proportion of cells that had periodic activity on each tetrode, including all tetrodes with at least four isolatable units. $\boldsymbol{h}$, Number of trajectory pairs with periodic firing per cell. $\boldsymbol{i}$, Distribution of normalized overlaps between the linearized firing of the same cell (black) on two different trajectories or different cells (light gray) on two different trajectories. The median for single cells was larger than for different cells ${ }^{* * *} p<10^{-10}$, rank-sum test). A diagram illustrating the normalized overlap measure is shown to the left. The linearized firing on each trajectory (top, a single cell's firing on one trajectory in pink and on another trajectory in purple) is divided by its area to produce firing rate curves with the same total firing (bottom). The normalized overlap is the area under both curves that are normalized by their area (gray in bottom graph). Only correct trials were included, and only trajectories with the same turn direction were compared. Results were similar if all trials were included. and 197 trajectory pairs for the same trajectories in the same sequence or the same trajectory in S1 and S2, respectively). Thus, switching from S1 to S2 does not appear to alter the coding for the shared central trajectory. Given that cells were most often path equivalent on two or fewer trajectory pairs (Fig. $3 h$ ), our results suggest that path equivalence of some cells could develop independently in S1 and S2.

Furthermore, these changes in path equivalence could not be explained by changes in place field properties related to the animal's behavioral performance. We compared periods when the animals performed the rewarded sequence with low or high accuracy; e.g., before and after the animals performed the rewarded sequence significantly more accurately than the unrewarded sequence (see Materials and Methods). We found no significant differences in several place field properties when the animal performed the rewarded sequence with low or high accuracy: spatial information ( $p>0.08$; medians, 2.9465 and 2.8088, respectively; $n=26$ and 64 trajectories for periods with low and high accuracy, respectively), mean firing rates $(p>0.56$; medians, 0.2736 and $0.3180 ; n=82$ and 90 cells for periods with low and high accuracy, respectively), and peak firing rates $(p>0.40$; medians, 6.2767 and 7.8427, respectively; $n=74$ and 90 cells for periods with low and high accuracy, respectively).

These findings indicate that path equivalence increases with experience with behaviorally related trajectories. That raises the possibility that the coding for the nonrewarded trajectories involving the outside arms of the maze would become less similar to the coding for the rewarded trajectories with experience. Although this may have occurred, animals stopped sampling the unrewarded outer arms of the maze during the initial learning, so we could not evaluate the similarity of coding across rewarded and nonrewarded arms.

\section{Ensembles of cells are recruited together in the path-equivalent code}

Previous studies have identified "cell assemblies," where subsets of neurons fire together more than expected (Harris et al., 2003; Dragoi and Buzsaki, 2006). The role of these ensembles in encoding specific mnemonic information remains unclear, however. We therefore asked whether path-equivalent neurons were active in the context of organized ensembles. If so, instead of single cells independently firing similarly across places, ensembles of cells could fire together in similar patterns across related places. Indeed, we found that path-equivalent cells that repeat together on multiple trajectories have correlated moment-to-moment activity, suggesting they are connected or receive similar inputs. These results could not be explained by a simple relationship between place field overlap and correlated moment-to-moment activity, more time bins available in cells pairs with higher overlap, or clustering errors.

To identify elements of cell ensembles, we examined correlations between pairs of cells' moment-to-moment variability. High correlations between cells' moment-to-moment variability suggest the cells receive similar inputs or are connected to each other either directly or indirectly (Lee et al., 1998; Shadlen and Newsome, 1998). We calculated the difference between each cell's expected number of spikes (based on linearized firing rates and the animal's position) and the actual number of spikes in 500 ms time bins (see Materials and Methods) (Fig. $5 a$ ). We then correlated these residuals between pairs of cells both in single trajectories and over the entire session when animals were running. Higher correlations indicate that the cells vary together from moment to moment. 

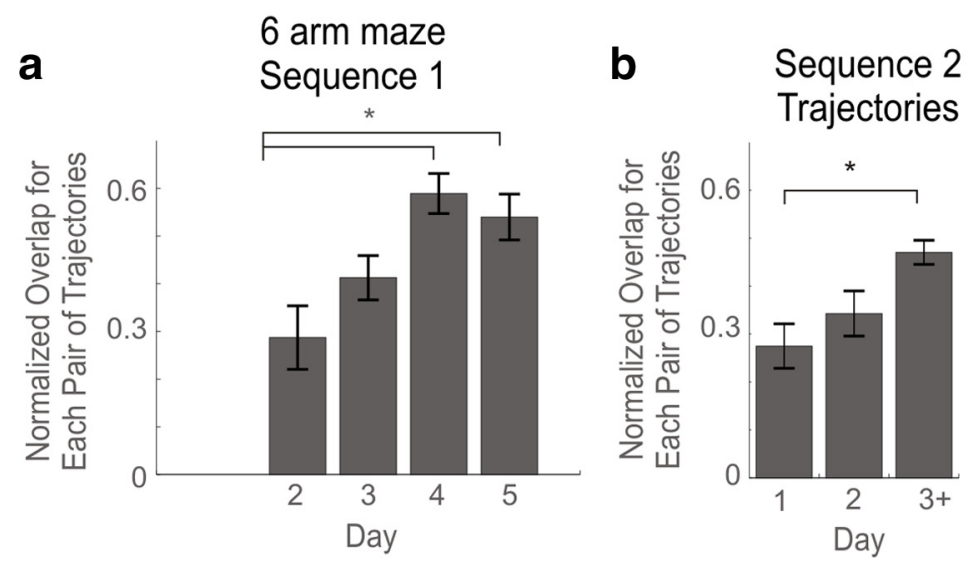

\section{6 arm maze Sequence 2}
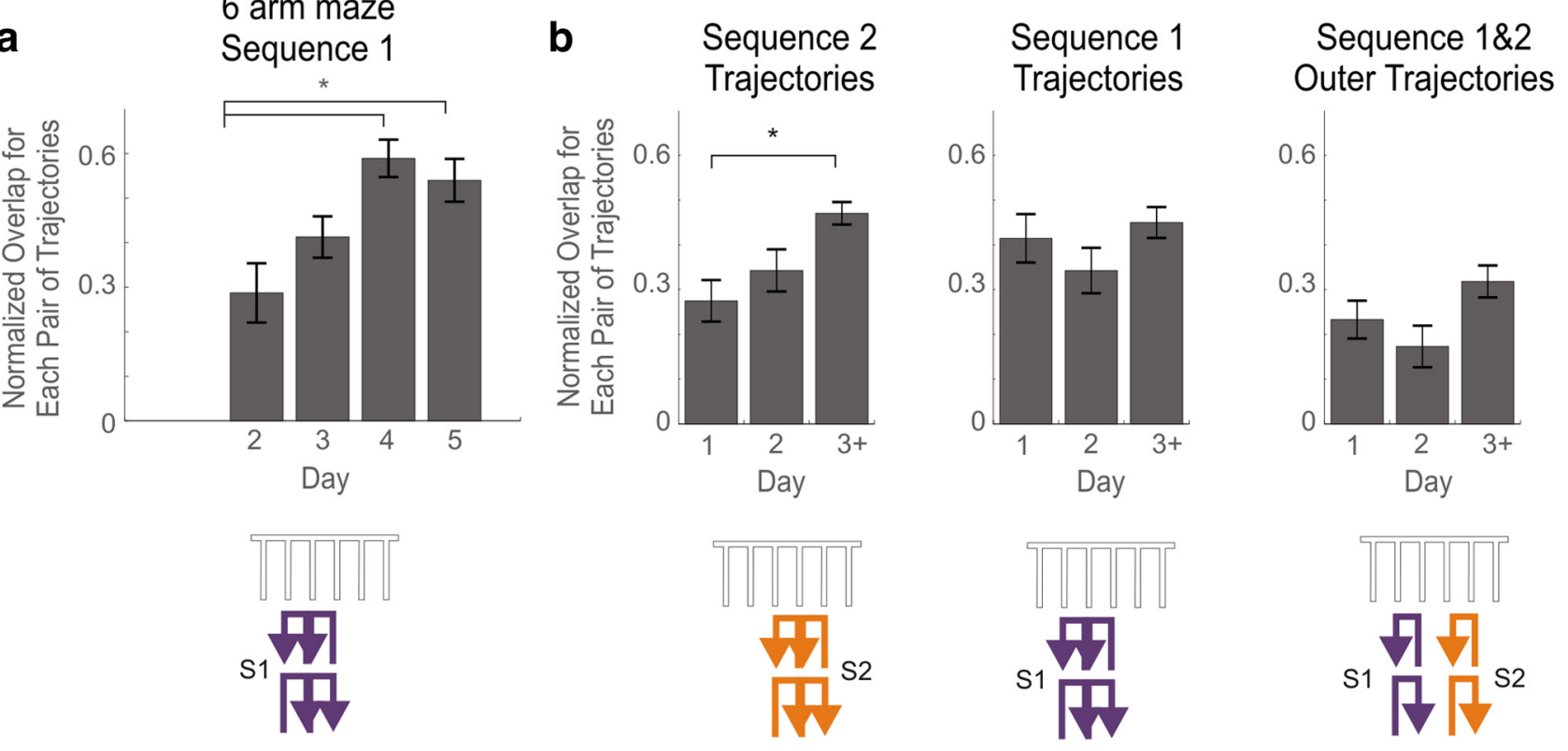

C
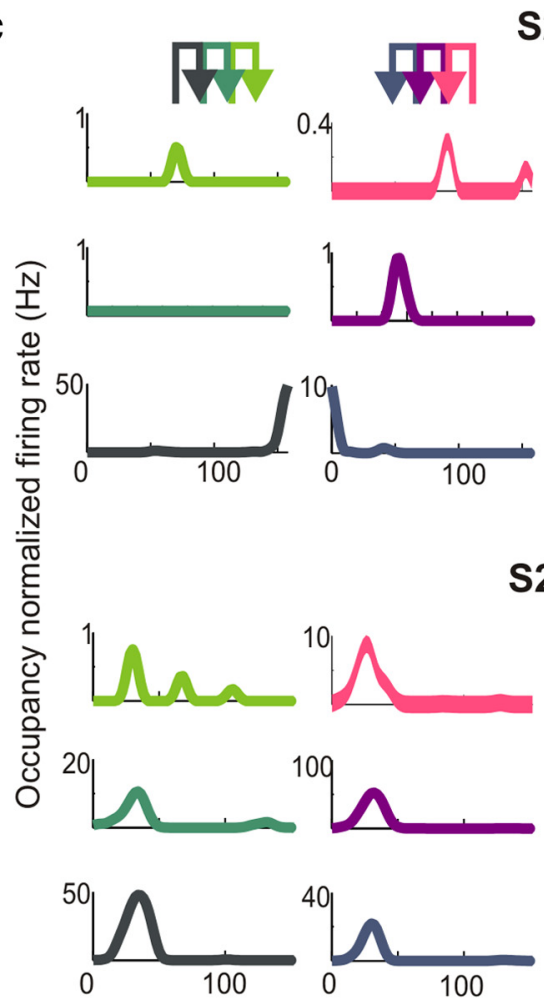

S2 Day 1
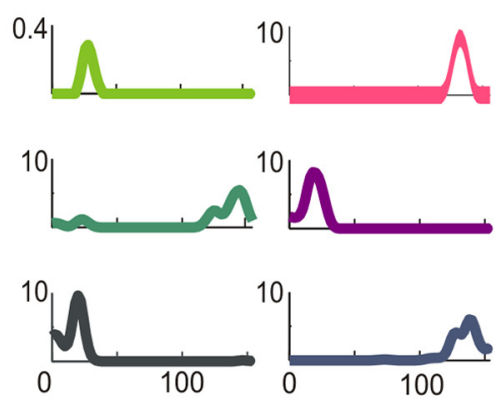

S2 Day 3

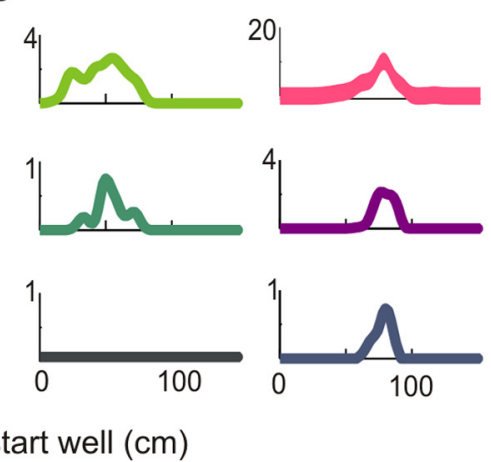

d

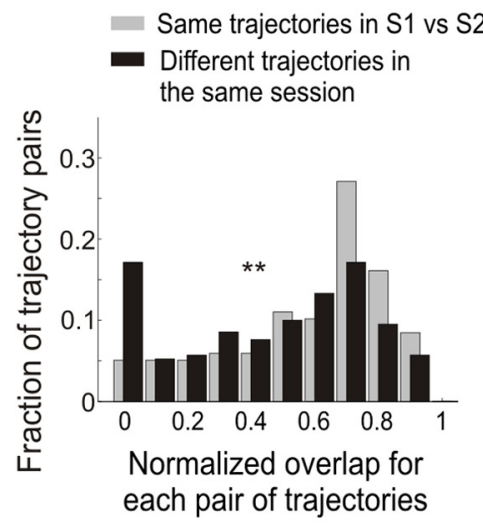

Distance from start well $(\mathrm{cm})$

Figure 4. Path equivalence increases with experience. $\boldsymbol{a}$, Normalized overlaps per trajectory pair during initial training on $\$ 1$ in the six-arm maze across days $2-5$ for trajectories that are part of $\$ 1$. The median normalized overlap increased for $\$ 1$ in the six-arm maze from day 2 to day 5 (Kruskal-Wallis one-way AN0VA, $\chi_{(4,115)}^{2}=17.46 ; p<0.005 ;$ the value for day 2 was significantly less than the values on days 4 and $5 ; p<0.05$, Tukey-Kramer post hoc test). There were insufficient data to compute the normalized overlap on day $1 . \boldsymbol{b}$, Normalized overlaps during training on $\$ 2$ during the switching phase of the task in the six-arm maze after initial training on S1. Normalized overlaps were examined for different pairs of trajectories, including trajectory pairs within S2, trajectory pairs within S1, and trajectory pairs where one trajectory was rewarded in S1 and the other in S2. 0nly trajectory pairs within S2 showed a significant change. Error bars show mean \pm SEM. c, Examples of firing on task-relevant trajectories of four different cells during training on S2. The plots depict the occupancynormalized linear firing rates for two cells taken from day 1 (above) and two from day 3 (below) of exposure to S2. $\boldsymbol{d}$, Distribution of normalized overlaps between the linearized firing of the same cell on the same trajectories when the animal performed $\$ 1$ or $\$ 2$ (light gray) or the same cell on different trajectories when the animal performed the same sequence in the same session (black). The median for same trajectories in S1 versus $\$ 2$ was larger than for different trajectories in the same session ( $p<0.001$, rank-sum test). ${ }^{*} p<0.05 ;{ }^{* *} p<0.001$. 

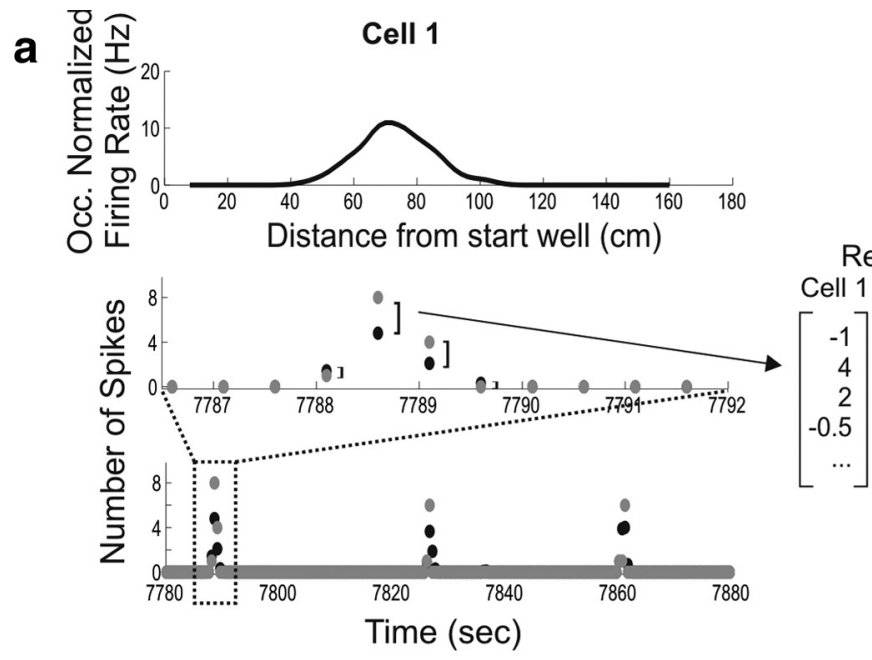

Residuals

Cell 2

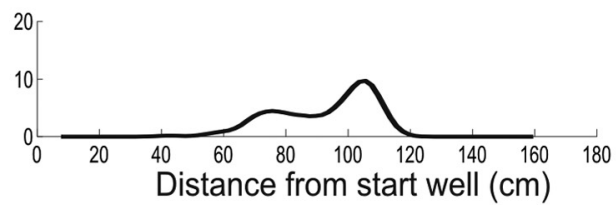

1 Cell 2

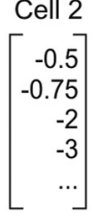

\section{b}

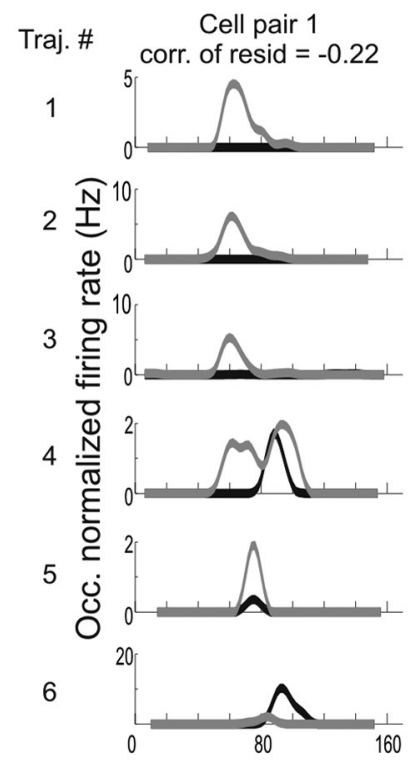

Cell pair 2

corr. of resid $=-0.15$

20

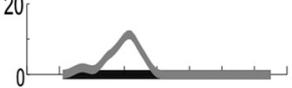

20
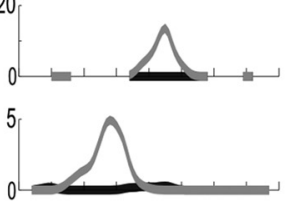

10
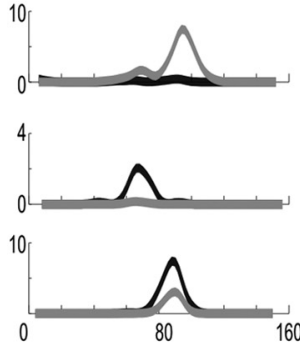

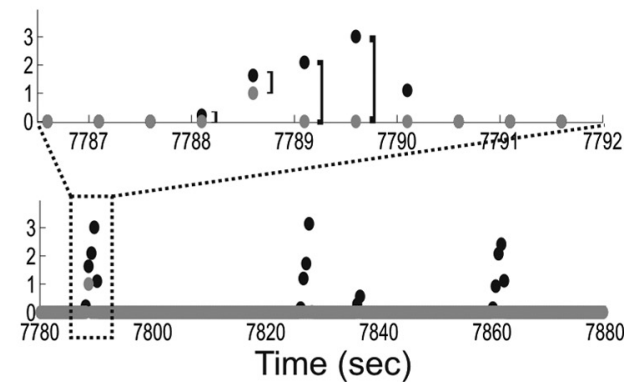

Cell pair 3

corr. of resid $=0.14$

10
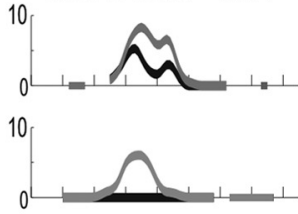

10
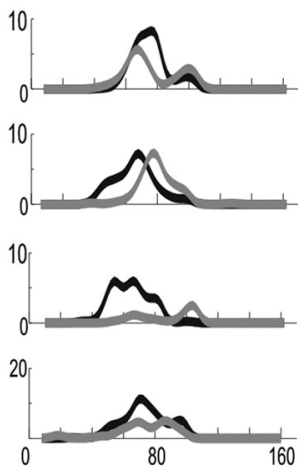

Cell pair 4

corr. of resid $=0.21$

Distance from start well $(\mathrm{cm})$
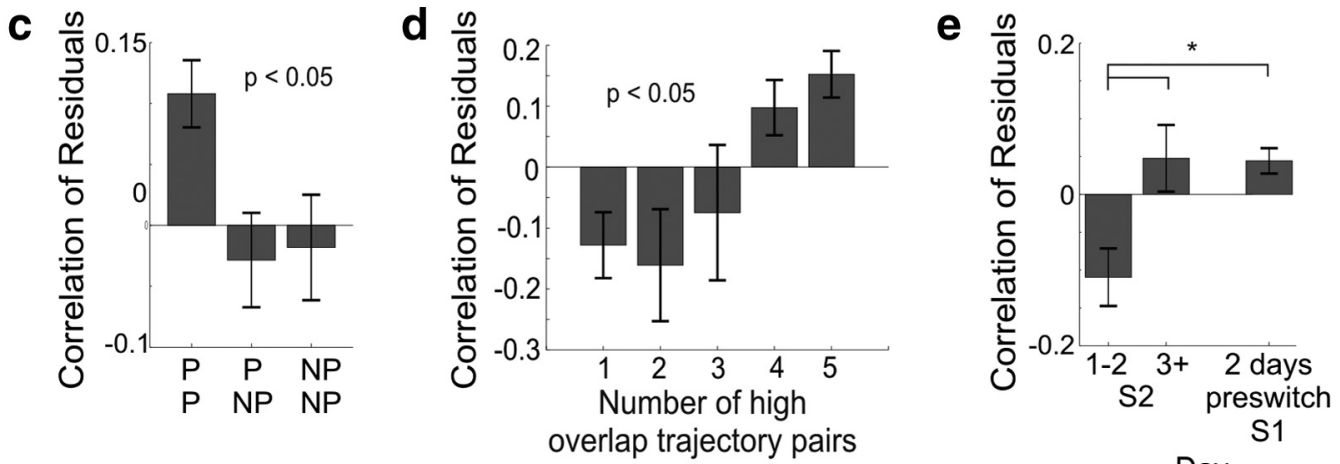

Day

Figure 5. Pairs of path-equivalent cells are correlated. $\boldsymbol{a}$, The linearized firing rate on a trajectory (top) was used to compute the expected number of spikes (black, middle, and bottom graphs) in each time bin for a cell (two example cells shown here). Each dot shows the expected (black) or actual (light gray) number of spikes in each time bin for a single pass through the trajectory (middle) and through three passes (bottom). The residuals are the difference between the expected and actual number of spikes in each time bin. $\boldsymbol{b}$, Examples of linearized firing curves on all task trajectories for different pairs of cells. Cell pairs 1 and 2 have negative residual correlations of -0.22 and -0.15 , respectively, and have low overlap in most trajectories. Cell pairs 3 and 4 have positive residual correlations of 0.14 and 0.21 , respectively, and have high overlap in most trajectories. Some bins of the linearized firing curves were excluded because of low occupancy (see Materials and Methods). c, Residual correlations for cell pairs in which both cells were path equivalent (P/P), one cell was path equivalent and one cell was not (P/NP), or both cells were not path equivalent (NP/NP). Residual correlations were significantly higher for pairs in which both cells were path equivalent (ANOVA, $p<0.05 ; F=3.32$ ). $\boldsymbol{d}$, The number of trajectory pairs with high overlap (overlap of $>0.3$ ) versus the correlation coefficient of the residuals for all cell pairs (ANOVA, $p<0.05 ; F=2.9704$ ). When comparing different trajectories, only trajectories in the same turn direction were compared. $\boldsymbol{e}$, Correlation of residuals during the first two days of training on S2 (left bar), during three or more days of training on S2 (middle bar) after initial training on S1, and during the last (Figure legend continues.) 
We found that pairs of path-equivalent cells had higher residual correlations than other pairs of cells, suggesting that pathequivalent cells are part of functional ensembles. Cells were categorized as path equivalent based on the autocorrelation analysis described above. We examined pairs of path-equivalent cells whose linear firing rate curves overlapped (overlap $>0.3$ ) in different numbers of trajectories. Pairs of path-equivalent cells that overlap together in multiple trajectories (three or more) had much higher residual correlations than pairs in which one or both cells were not path equivalent (Fig. 5b,c; supplemental Fig. 3, available at www.jneurosci.org as supplemental material) (ANOVA, $\left.F_{(2,60)}=3.32 ; p<0.05\right)$. We found the same trend for pairs of path-equivalent cells that overlapped in two or more trajectories but the effect was not significant (ANOVA, $F_{(2,63)}=$ $1.857 ; p<0.17)$. Although we might expect that pairs of nonpath-equivalent cells would also form functional ensembles, we did not find any evidence for that here. This is perhaps not surprising as cells with overlapping place fields in a single location could have similar field locations by chance, and thus might not be part of an ensemble.

Furthermore, we found that the larger the number of trajectories in which two cells' linearized firing curves overlapped, the more correlated their moment-to-moment activity (Fig. $5 d$ ) $\left(\right.$ ANOVA, $\left.F_{(4,30)}=2.9704 ; p<0.05\right)$ (see Materials and Methods). We noted that the pairs with overlap in two or fewer trajectories tended to be negatively correlated, perhaps reflecting a mechanism that maintains low or negative correlations among less related neurons. We also examined the distributions of the correlations of residuals between all cell pairs and pairs with low or high overlap (supplemental Fig. $4 a-c$, available at www. jneurosci.org as supplemental material). Cell pairs with overlapping fields (overlap of $>0.3$ ) in at least three trajectories (high overlap) had higher residual correlations than cell pairs with low overlap (overlap of $<0.2$ ) in at least three trajectories $(p<0.0005)$. Consistent with these results, we found that cell pairs with overlapping place fields and correlated moment-tomoment activity in one location are more likely to have overlapping place fields in another location (supplemental Fig. $4 d$, available at www.jneurosci.org as supplemental material). Only cells with a minimum total overlap across all trajectories of at least 0.1 were included for these analyses; cell pairs could overlap on a single trajectory or on multiple trajectories. Although this excluded many cell pairs, it ensured that the pairs that were included had some overlapping fields on at least one trajectory on which to compute the residual correlation.

In addition, the correlations of residuals increased as animals gained more experience in S2. Given that overlapping, pathequivalent cells were correlated and that path equivalence increased with experience, we would expect that these correlations would also increase with experience. Including all cell pairs with an overlap of at least 0.1 across all trajectories, we computed the correlation of residuals for the entire session when animals ran faster than $3 \mathrm{~cm} / \mathrm{s}$ and performed $\mathrm{S} 2$ on the first two days of exposure to $\mathrm{S} 2$ or three or more days of exposure. We grouped days to include more data in each group. We also compared this

$\leftarrow$

(Figure legend continued.) two days of training on S1 before the switching phase and training on S2 commenced (right bar). Correlations increased from days 1-2 to days 3 or more (ANOVA, $F_{(2,115)}=5.9582 ; p<0.005 ; \mathrm{S} 2$ days 1 and 2 was significantly smaller than $\mathrm{S} 3$ day $3+$ and $\mathrm{S} 1$ $2 \mathrm{~d}$ preswitch, $p<0.05$, Tukey-Kramer post hoc test). Only cell pairs with an overlap of at least 0.1 across all trajectories were included. Error bars show mean $\pm \mathrm{SEM} ;{ }^{*} p<0.05$. to the last two days of exposure to S1 during the initial training on S1 before the sequence-switching phase of the task began. We found that the residual correlations were higher when animals had three or more days of exposure to the sequence than on days 1 and 2 (Fig. 5e) (ANOVA, $F_{(2,115)}=5.9582 ; p<0.005$; medians, $-0.1117,0.0630$, and 0.0348 for S2 days 1 and 2, days $3+$, and S1 last $2 \mathrm{~d}$ preswitch, respectively; S2 days 1 and 2 median is significantly less than that for S2 days $3+$ and S1 last $2 \mathrm{~d}$ preswitch; $p<$ 0.05 , Tukey-Kramer post hoc test). The same trends were evident when we examined different types of cell pairs with different combinations of path-equivalent and non-path-equivalent cells, but the comparisons were no longer significant (data not shown). We also examined the correlations associated with the first few days of experience on S1, but found no clear trends, perhaps because place fields were still forming during this period (Leutgeb et al., 2004; Karlsson and Frank, 2008).

We then performed four important control analyses. First, we asked whether high correlations were a general feature of place fields with high overlap. We found that this was not a likely explanation, because there was no significant relationship between the correlations of residuals and overlap in the same trajectory for cells with a peak firing rate of at least $0.5 \mathrm{~Hz}$ in the trajectory (supplemental Fig. $4 e$, available at www.jneurosci.org as supplemental material $)(\rho=0.0456 ; p>0.7 ; n=729$ trajectories $)$. We also examined the relationship between overlap and the residual correlation only in cases with overlap $>0.3$, because we noticed that the distribution of the correlations seemed qualitatively different at overlaps above and below 0.3. Again, we found no significant relationship between the residual correlations and overlap in the same trajectory $(\rho=0.1273 ; p>0.18 ; n=174$ trajectories). These results indicate that there is no relationship between place field overlap and the correlation of residuals within a single trajectory. Instead, this relationship is only evident when we examine path-equivalent pairs across multiple trajectories.

Second, we determined that the relationship between overlap and correlation of residuals was not attributable to the greater number of time bins available for analyses in cells with high overlap. The larger the overlap between linearized firing curves, the greater the number of time bins in which we could compute a meaningful residual correlation. If cell pairs with little overlap had lower residual correlation simply because of fewer available time bins, we would expect a positive relationship between the number of time bins and the residual correlation value. Again we only examined cell pairs with an overlap of at least 0.1 across all trajectories, though we found similar results if we included all cell pairs. Because we would expect to see this relationship with few time bins, we examined this relationship for cases with 20 (the minimum allowed number of time bins) to 100 time bins. We found no significant relationship between the number of time bins and the residual correlation (supplemental Fig. $4 f$, available at www.jneurosci.org as supplemental material) $(\rho=0.12102$; $p>0.37 ; n=97$ cells). We conclude that correlation in a cell pair's moment-to-moment variability was not attributable to measurement error or bias, but instead reflects the short-timescale covariation of the cell pairs.

Third, we established that the higher residual correlations were not explained by higher total firing in path-equivalent cells. We computed the total firing over all trajectories for each cell as the area under the linearized firing rate curves. For each trajectory pair, we took the average of both cells' total firing and compared it to the cell pairs' residual correlation. We found no significant relation between the average total firing and the correlation of residuals (supplemental Fig. $4 g$, available at www.jneurosci.org as 
supplemental material) $(\rho=0.16234 ; p>$ $0.19 ; n=90$ cell pairs). We found similar results if we examined the minimum or maximum total firing of the pair, or if we examined the peak firing rates of the pair. Therefore, the higher total firing of pathequivalent cells could not explain their higher residual correlations.

Finally, to control for possible contamination attributable to clustering errors (Quirk and Wilson, 1999), we repeated all of these residual correlation analyses for cells recorded on different tetrodes. We found similar results for all of the analyses of residual correlations described above except one, in that the relationship between cell pairs with larger numbers of trajectories with overlapping fields still tended to have higher correlations of residuals for each cell pair, but the results were no longer significant (ANOVA, $\left.F_{(4,13)}=0.66 ; p=0.6298\right)$. This is not surprising because restricting the analyses to cells on different tetrodes reduced the number of cell pairs substantially. Because all of the other findings were consistent with our previous results, we believe the lack of significance in this case is attributable to the reduced number of cell pairs.

We also obtained the same residual correlation results when we included only every other day of data to help control for possible repeat recordings of the same cell on multiple days. As for the normalized overlap measure, we examined the relationship between cell pairs' residual correlations and the number trajectories with high overlap in linearized firing. Again, we found that the larger the number of trajectories in which two cells' linearized firing curves overlapped, the more correlated their moment-to-moment activity $\left(\right.$ ANOVA, $F_{(4,87)}=2.39 ; p<0.06$ ) (see Materials and Methods). These results approached significance, and when we grouped the data into pairs with less than three or three or more trajectories with high overlap, we found that the pairs with three or more highly overlapping trajectories had much higher residual correlations $(p<0.005$; medians, -0.0126 and 0.1202 for cell pairs with less than three or three or more trajectories with high overlap, respectively; $n=76$ and 16, respectively). Thus, our results remain significant with substantial reductions in the total numbers of cells analyzed when controlling for potential clustering errors or cells recorded on multiple days.

\section{Differences in firing rate and population coding associated with path equivalence}

The above analyses suggest that path equivalence could encode similarities across distinct locations, providing a framework to form common behavioral associations with multiple places. At the same time, animals would still need to distinguish among these places to perform spatial tasks. We therefore examined population activity and found that the population as a whole could $10^{-5} ;{ }^{* *} p<0.0001 ;{ }^{*} p<0.02$. b
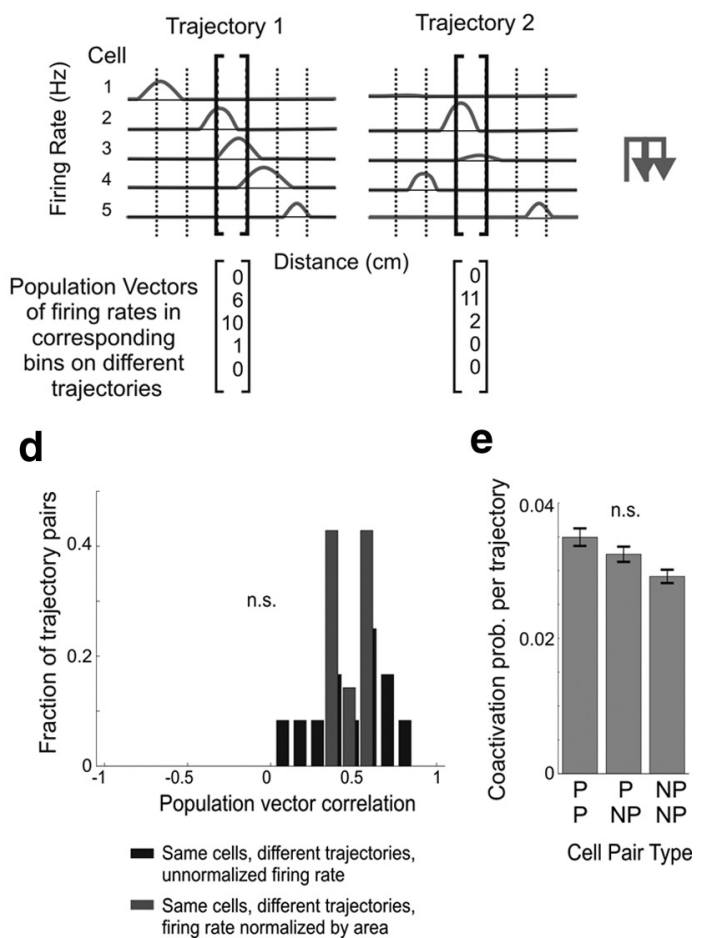

Figure 6. Firing rate differences create distinct population code. $\boldsymbol{a}$, Histogram of peak rate ratio of cell pairs' linearized firing comparing different trajectories with similar firing locations in the same run session (black; defined as normalized overlap of $>0.3$ )

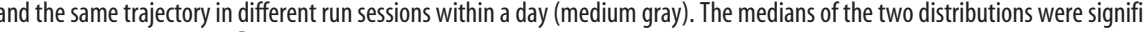
,. $\mathbf{b}$, To compare population level activity across trajectories, population vectors were constructed. Each the resulting correlations from all bins in a pair of trajectories were averaged. of population vector correlation of different trajectories in the same session (black), the same trajectory in different comparing different trajectories only trajectories in the same turn direction were compared. $\boldsymbol{d}$, Histogram of population vector correlation of different trajectories in the same session with linearized firing either normalized by its area to reduce differences in (black). The distributions were not significantly different $(p>0.7)$.e, Coactivation probability path-equivalent cells as other non-path-equivalent cells and vice versa. n.s., Not significant. Error bars show mean \pm SEM; ${ }^{* * *} p<$

distinguish between the arms of the six-arm environment despite the path-equivalent coding of individual cells. We first noted that although many cells fired in very similar locations in different trajectories in a repetitive environment, they fired at different peak rates, similar to reports of rate remapping (Leutgeb et al., 2005b). We compared peak linearized firing rates between two trajectories when the firing on those trajectories was in similar locations (normalized overlap of $>0.3$ ) (see Materials and Methods). We took a ratio of the smaller peak divided by the larger peak firing rate. We found the peak rate ratio between different trajectories with overlapping firing locations was lower than if we compared the peak firing on the same trajectory in different run sessions on the same day (Fig. $6 a)\left(p<10^{-5}\right.$; medians, 0.3695 and 0.6072 , respectively; $n=150$ and 197 trajectory pairs for different trajectories in the same session or the same trajectory in different sessions, respectively). We found similar results if we used a higher normalized overlap requirement to select cells with similar firing locations in different trajec- 
tories. Thus, cells that fire in similar locations in different trajectories fire at different peak rates.

Together, cells with path-equivalent coding but differences in peak firing rate and cells without path-equivalent coding generate distinct population codes in each trajectory. To examine this issue, we compared population activity in different trajectories by computing a population vector of firing rates of multiple single cells in each $10 \mathrm{~cm}$ bin of each trajectory (Fig. $6 b$ ). We then correlated vectors of corresponding bins from trajectories in the same turn direction and averaged the correlations for each trajectory. We found the resulting population vector correlations were significantly lower than if we compared the same trajectory in different sessions (Fig. $6 c)(p<0.02 ; n=10$ averaged trajectory pairs), revealing significant differences between trajectories in the population code. The population vector correlations were also higher than if the cell identity was shuffled, revealing that the repetitive coding structure in some cells does result in higher correlation in the population activity (Fig. $6 c)(p<0.0001 ; n=$ 10 averaged trajectory pairs). Because, by definition, non-pathequivalent cells fire differently on different trajectories, shuffling the cell identity is similar to examining populations without path-equivalent cells. This suggests that a population with both path-equivalent and non-path-equivalent coding may simultaneously generalize and separate.

To determine the relative contribution of rate remapping in path-equivalent cells to the population code, we also computed the population vector correlations using the normalized linearized firing rates. With the normalized linearized firing, each cell has the same total firing on each trajectory, reducing differences in activity attributable to rate remapping. We found that the distribution of population vector correlations from normalized linearized firing was similar to the correlation from nonnormalized firing, though the distribution was narrower (Fig. $6 d)(p>0.7$; medians, 0.5922 and 0.5103 for nonnormalized and normalized, respectively; $n=10$ averaged trajectory pairs). The population vector correlations from normalized firing were still significantly lower than if we compared the same trajectory in different sessions $(p<$ $0.001 ; n=10$ averaged trajectory pairs), and higher than if the cell identity was shuffled ( $p<0.001 ; n=10$ averaged trajectory pairs). These results indicate that even without extensive rate remapping, a population of path-equivalent and non-path-equivalent cells can create distinct population codes for different trajectories.

These findings suggest that CA3 population activity contains information representing the similarities across locations and information about each individual location. We then asked whether these two types of information are present simultaneously or whether the system alternates between path-equivalent and non-path-equivalent representations. We found that both path-equivalent and non-pathequivalent cells fire together on short time scales. We examined the probability of two cells firing together in $100 \mathrm{~ms}$ bins while animals were running on each trajectory for pairs of path-equivalent cells (as determined by the autocorrelation analyses above), pairs with one path-equivalent and one non-path-equivalent cell, and pairs of nonpath-equivalent cells. We computed the coactivation probability per trajectory to avoid biases caused by the fact that pairs of periodic cells have more place fields across multiple trajectories. We chose $100 \mathrm{~ms}$ bins because this is about the length of a theta cycle, which is often considered an important computational unit.

We found that per trajectory, path-equivalent cells were equally likely to fire with non-path-equivalent and pathequivalent cells (Fig. 6e) ( $p>0.5$; medians, 0.0296, 0.0294, and 0.0276 for path-equivalent pairs, path-equivalent/non-pathequivalent pairs, and non-path-equivalent pairs, respectively; $n=235,209$, and 167 cell pairs, respectively). Although there was a trend for pairs of non-path-equivalent cells to fire together less than pairs of periodic cells, this result was not significant when taking into account the multiple comparisons made (Fig. 6e). We also examined the coactivation probability per trajectory in $25 \mathrm{~ms}$ bins, or about the length of a gamma cycle, and found similar results (medians, 0.0067, 0.0069, and 0.0063; $p>0.5$, rank-sum test of coactivation probability of path-equivalent pairs and pairs of path-equivalent and non-path-equivalent pairs) (data not shown). Together, these results indicate that the hippocampus can simultaneously represent similarities across locations and unique information about each location.

\section{Path equivalence is less common in the three-arm maze}

If similar coding patterns reflect learned generalizations, then repetitive coding would be less common in a simpler, less repetitive environment. Indeed, a previous report has indicated that path equivalence was not prevalent in CA1 cells recorded while animals performed a spatial alternation task on a three-arm "Wtrack" maze (Frank et al., 2000). The environment in that study was highly familiar, and no CA3 cells were sampled, however. Since previous studies have reported differences between CA3 and CA1 (Guzowski et al., 2004; Leutgeb et al., 2004, 2005a; Lee et al., 2004), we wondered whether path-equivalent activity is absent in a three-arm maze or if it only occurs in CA3 or during learning in such a maze. We therefore examined three-arm maze data from both CA3 and CA1 cells (Karlsson and Frank, 2008, 2009). The alternation task in the three-arm maze was the same as $\mathrm{S} 1$ or S2 in the six-arm maze; however, there were fewer arms, and none of the arms were exactly geometrically identical (Fig. 2). In particular, in the six-arm maze, all the arms end in a $\mathrm{T}$ junction, whereas in the three-arm maze the middle arm ends in a $\mathrm{T}$, and the left and right arms end in right-turn-only or left-turn-only $\mathrm{L}$ shapes.

We found that most cells had single place fields in the threearm environment (Fig. 7a,b, left, right), though some had multiple fields in somewhat similar locations in different arms (Fig. $7 a, b$, middle). Cells that had a single place field could fire in a single trajectory (Fig. $7 a, b$, left) or multiple trajectories as the animal traversed the same location in multiple trajectories (Fig. $7 a, b$, right). However, these cells generally did not have peaks in the autocorrelogram at the length of a single trajectory $(\sim 180$ $\mathrm{cm}$ ), as we would expect if the cells' firing was periodic (Fig. 7c). Across the population, both CA3 and CA1 cells with multiple place fields had some tendency to be active at lags of about the length of a trajectory (Fig. $8 a, d$ ), but the overall proportion of periodic cells $(160-200 \mathrm{~cm})$ was lower than in the six-arm maze (Fig. $8 b, e)\left(p<0.05, \chi^{2}\right.$ test for both CA3 and CA1 cells from the three-arm maze compared to CA3 cells in the six-arm maze).

Similarly, in the three-arm maze, the spatial locations of firing from the same cell on different trajectories were more similar to firing from different cells on different trajectories. Unlike in the six-arm maze, the distribution of normalized overlaps was not bimodal, but instead decreased monotonically from 0 to 1 (Fig. $8 c, f)$. The same and different cell distributions did have slightly different medians (CA3: medians, 0.1697 and $0.1211 ; n=260$ and 1692 same cell and different cell distributions, respectively; $p<0.05 ;$ CA1: medians $=0.1557$ and $0.1129 ; n=309$ and 2659 same cell and different cell distributions, respectively; $p<0.005$ ), but the CA3 same cell distribution from the three-arm maze had a much lower normalized overlap than that from the six-arm maze (median, 0.5574 for six-arm maze same cell; $p<10^{-5}$ ). We conclude that path-equivalent coding in CA3 and CA1 is present, 
a

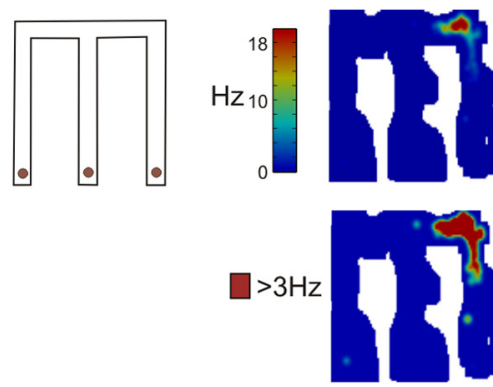

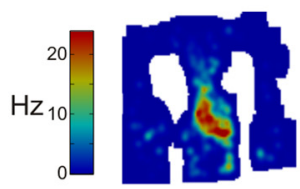

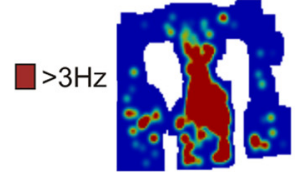

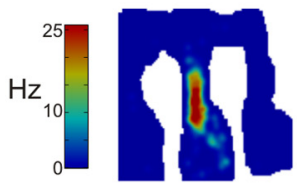

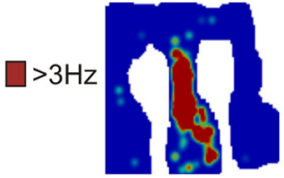

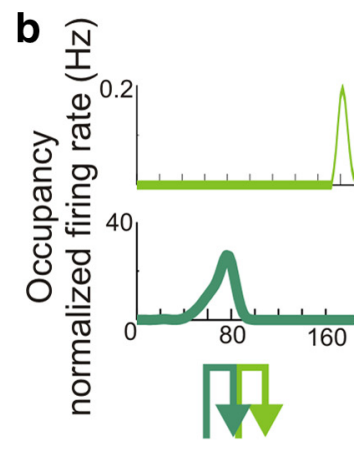

C

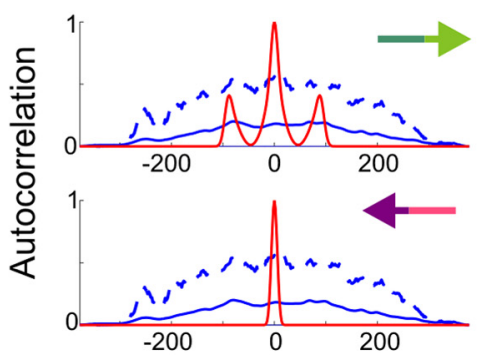

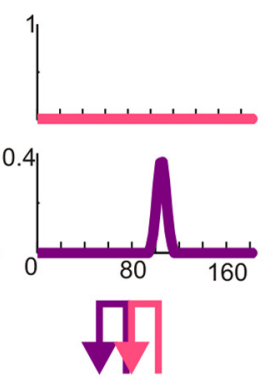
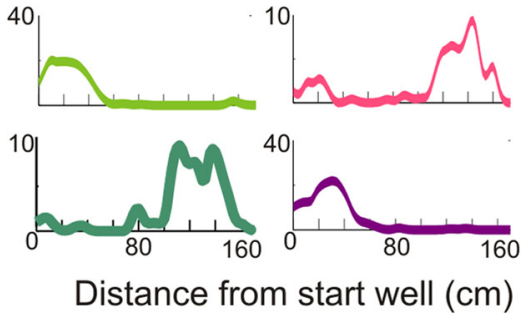

Distance from start well $(\mathrm{cm})$
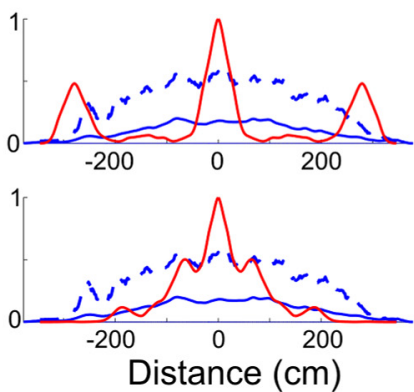
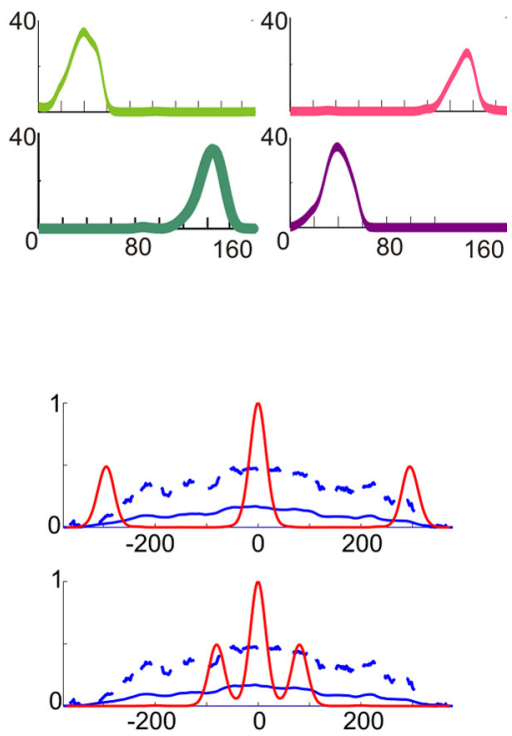

Figure 7. Path-equivalent coding is less common in the three-arm maze. $\boldsymbol{a}$, Overhead view of the three-arm maze (top left) and spatial firing rate maps of three cells scaled to the cells' peak firing rate (above) or scaled so that all firing of $>3 \mathrm{~Hz}$ is red (below). The cells shown on the left and center were recorded in CA3, and the cell shown on the right was recorded in CA1. The cell shown in the center fired in several arms in some similar locations, whereas the other cells had place fields limited to a single arm. Only times when the animal was running $>3 \mathrm{~cm} / \mathrm{s}$ were included. $\boldsymbol{b}$, 0ccupancy-normalized firing rates on each linearized trajectory for the same three cells shown in $\boldsymbol{a}$. $\boldsymbol{c}$, Autocorrelation of the linearized firing for the same three single cells shown in $\boldsymbol{a}$ and $\boldsymbol{b}$ (red) and the mean cross-correlation of the linearized firing between different cells (blue; solid line, mean; dashed line, $90 \%$ confidence bounds). Linearized firing rates on trajectories in the same turn direction were normalized by their area and then concatenated. The top shows right-turn trajectories as the animal moves from the left to the right, and the bottom shows left turns as the animal moves from the right to the left.

but much less common in the three-arm maze compared to the six-arm maze, even though the animals were executing the same alternation task. There were no differences in the prevalence of path-equivalent coding between CA3 and CA1.

Furthermore, in the three-arm maze, normalized overlap did not change with experience. We examined normalized overlap of CA3 cells between trajectory pairs in $\mathrm{T} 2$ in the three-arm maze, from the animals first exposure to $\mathrm{T} 2$ to the fifth day of exposure. We found no significant change in normalized overlap across days or between the first day of T2 exposure and exposures to the familiar track, track 1 (Fig. 8g) (Kruskal-Wallis one-way ANOVA, $\chi_{(4,108)}^{2}=7.8749 ; p>0.09$; medians, 0.3454, 0.3146, $0.2682,0.2333$, and 0.1636 for days $1-5$ on T2; no significant differences between days $1-5$ on T1; $p>0.05$, Tukey-Kramer post hoc test). In fact, the distributions of normalized overlap values for the highly familiar T2 were similar to those of the novel six-arm maze (Fig. $8 h$ ), indicating that both environments began with comparable overlaps across trajectories. Thus, the overlaps increased with experience in the six-arm maze, but there were no changes over time in the three-arm maze.
Path equivalence in CA1 in multiple- $U$ environment

Finally, to better understand the role of geometric versus local cue-based similarity in path-equivalent activity, we examined CA1 neurons in two animals traversing a multiple- $U$ environment where each $U$ had different local visual and olfactory cues (Fig. 2b). We examined these parameters because previous studies have shown that subtle environmental manipulations can lead to distinct patterns of rate and global remapping (Skaggs and McNaughton, 1998; Lever et al., 2002; Leutgeb et al., 2004, $2005 \mathrm{a}, \mathrm{b})$. In the multiple-U environment, the local sensory cues differed substantially across each U (see Materials and Methods), but despite these differences, path-equivalent coding was common. We found many single cells fired in similar locations in the different U's that make up the track, whereas others only fired in a single location (Fig. 9a). Some cells fired around the corners and other cells fired along straight sections of the track, suggesting that this activity is similar to that seen in the six-arm maze.

As in the six-arm maze, we found that the place field firing locations on some, but not all, possible trajectories was highly similar. Only 24 cells reached the criteria for inclusion in the 

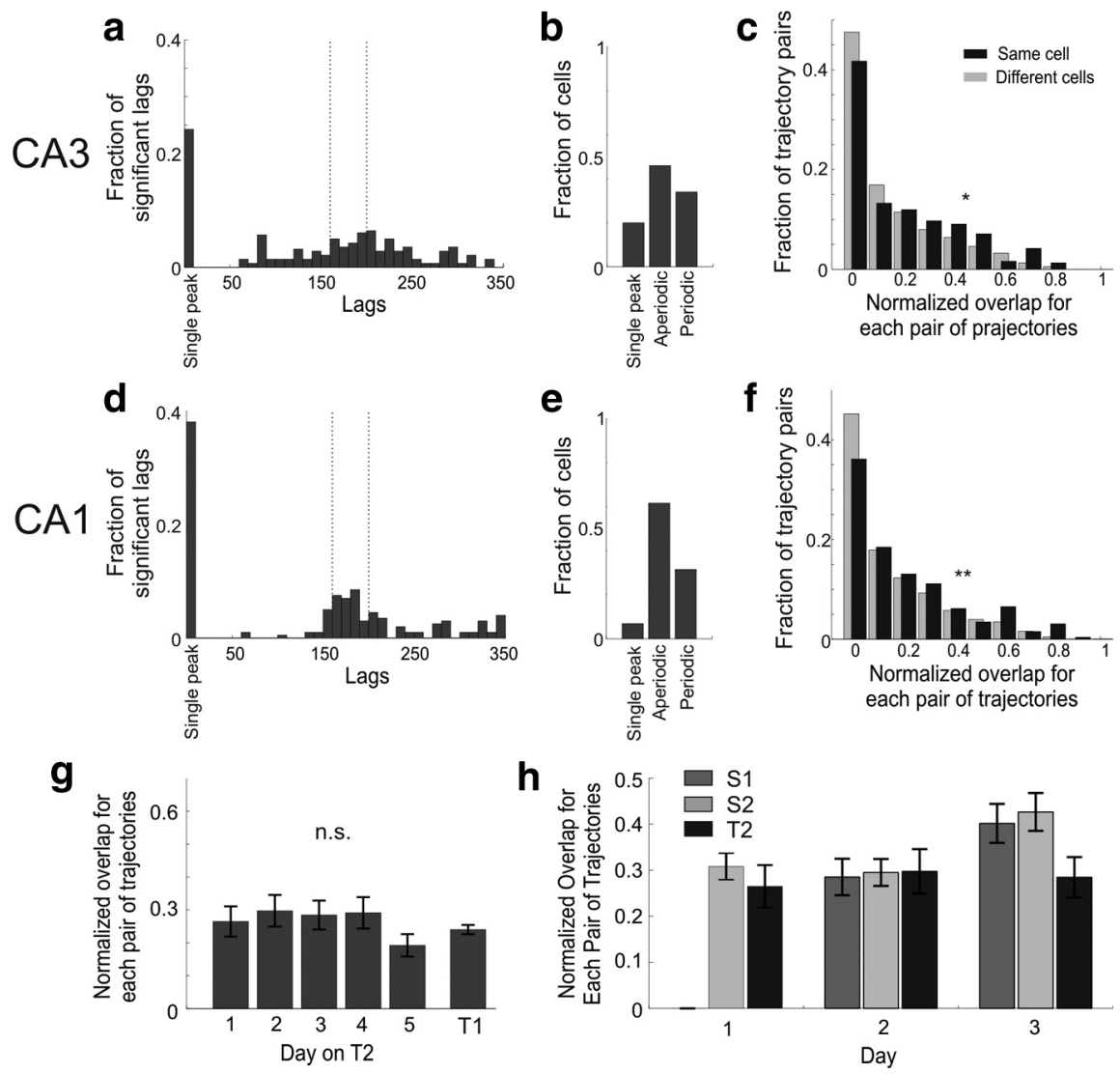

Figure 8. Quantification of path-equivalent activity in the three-arm maze. $\boldsymbol{a}$, Histogram of significant peaks at lags of $>60 \mathrm{~cm}$ for each cell's linearized firing autocorrelation in CA3. $\boldsymbol{b}$, Proportion of cells with periodic or aperiodic activity, or no significant peaks at lags $>60 \mathrm{~cm}$ in CA3 (single peak). c, Distribution of normalized overlaps between the linearized firing of the same cell (black) on two different trajectories or different cells (light gray) on two different trajectories in CA3. The median values were significantly different ( ${ }^{*} p<0.05$ ) but were similar in magnitude (medians: 0.1697 , same cell; 0.1211 , different cell). $\boldsymbol{d}-\boldsymbol{f}$, The same as $\boldsymbol{a}, \boldsymbol{b}$, and $\boldsymbol{c}$ for CA1. Once again, the median normalized overlap values were significantly different ( $\left.{ }^{* *} p<0.005\right)$ but were similar is magnitude (medians: 0.1557 , same cell; 0.1129 , different cell). Only correct trials were included, and only trajectories in the same turn direction were compared. Results were similar if all trials were included. $\boldsymbol{g}$, Normalized overlap in the initially novel three-arm maze, T2, and from T1. There were no significant changes in the distributions across days (Kruskal-Wallis one-way ANOVA, $\chi_{(4,108)}^{2}=7.8749 ; p>0.09$; the days were not significantly different; $p>0.05$, Tukey-Kramer post hoc test). n.S., Not significant. $\boldsymbol{h}$, Normalized overlap per trajectory pair for days 1 to 3 in $\mathbf{S 1}$ (dark gray) (4a) and S2 (light gray) (from Fig. 4b) in the six-arm maze and $\mathrm{T} 2$ in the three-arm maze (black) ( $\boldsymbol{g}$ ) for direct comparison. The similarities in the overlaps suggest that CA3 cells start out with more dissimilar firing across trajectories, but that as the animal learns the task in the six-arm maze, the coding for these trajectories becomes more similar. Error bars show mean \pm SEM.

analysis, making it difficult to apply the autocorrelation analysis used for the six-arm maze. As an alternative, we computed the normalized overlap between linearized firing from the same cell on different trajectories and from different cells on different trajectories. We took the maximums of the normalized overlaps for the same-cell and different-cell pairs. Cells tended to be path equivalent across a subset of the U's and were often not active at all in the other U's. Taking the maximum normalized overlap across all trajectory pairs highlighted the subset of U's in which the cell was active in both trajectories and path equivalent. The maximum normalized overlaps of all the trajectory pairs from the same cell had a bimodal distribution that was different from the distribution from different cells (Fig. 9b) (KomolgorovSmirnov test; $p<0.01$ ). The shape of this distribution suggests that, as in the six-arm maze, about half of the cells showed periodic, path-equivalent activity, whereas the other half do not.

Including normalized overlaps from all trajectory pairs, not just the maximum per cell, the normalized overlap between linearized firing from the same cell on different trajectories was also significantly higher than firing from different cells (Fig. 9c) $(p<0.001)$. These results establish that path equivalence also occurs in CA1, is not attributable to the specifics of the six-arm paradigm, and, because each $U$ had very different visual and olfactory cues, is not likely to be attributable solely to similarity of local sensory cues. This further supports our finding that path equivalence is not the result of an animal's failure to distinguish between similar locations.

\section{Discussion}

We found that hippocampal neurons fire in multiple similar locations in environments with repeated elements even when animals must behaviorally distinguish among the elements, and even when the elements have distinct visual and olfactory cues. This path-equivalent coding develops as animals learn the rewarded sequences and the relationships between paths. Furthermore, path-equivalent firing is not simply the result of single cells acting independently. Rather, pairs of cells whose activity varied together from moment to moment also repeated together in multiple segments, suggesting that ensembles of cells are recruited together. This correlated firing also became more prevalent with experience, pointing to a role for learning in the development of path-equivalent coding. These results are consistent with a functional role for hippocampal ensembles in coding for general features across locations (Frank et al., 2000). At the same time, the simultaneous presence of path-equivalent and non-path-equivalent coding on short time scales indicates that hippocampal cells can simultaneously represent both common elements across locations and the unique features of each location.

Path equivalence cannot be explained by confounding factors In previous reports of path equivalence and rate remapping, the functional significance of these coding schemes has been unclear, because they could have been explained by a number of confounding factors. In this study, we show that these patterns of activity cannot be explained by the animal's failure to distinguish among the arms, a lack of hippocampal involvement in the task, a dearth of differentiating sensory cues, or errors in isolating single cells. First, correct behavioral performance in the six-arm task requires that the animal make different choices depending on current and past locations. Although it is conceivable that the animal could make these choices without an accurate hippocampal representation of location, there is good reason to believe that the hippocampus is involved in learning this task. When animals were first placed in the home arm of S2, they immediately moved to the adjacent arm and performed the previously rewarded S1. This indicates that the animals were using an allocentric strategy associated with hippocampal dependence (Packard 
and McGaugh, 1996). In addition, this task is based on a the simpler W-track alternation task where the hippocampus is required for rapid learning (Kim and Frank, 2009). The six-arm task also requires flexible changes in behavior in response to changing reward contingencies. Because this sort of flexibility generally requires the hippocampus (Hsiao and Isaacson, 1971; Hirsh et al., 1978; Ainge et al., 2007), hippocampal activity is likely to be important for learning to switch between sequences. Thus, there is good reason to believe that the hippocampus was engaged during the performance of the sixarm task and that animals distinguished among the arms of the environment.

Second, the prevalence of pathequivalent activity in the multiple- $U$ track argues that this activity is not attributable to a lack of distinguishing sensory cues. Each U of that environment was bordered by walls with highly distinctive visual patterns and was scented uniquely. Given that animals can use local visual and odor cues to distinguish among locations (Anderson and Jeffery, 2003), it is unlikely that path-equivalent activity in the hippocampus was attributable solely to sensory similarity.

Finally, there is no reason to suspect that errors in clustering could have led to our findings. Clustering errors would lead to errors such that a single clustered "unit" would have multiple place fields corresponding to the place fields of the single cells that were erroneously com-

bined. However, fields from different single cells erroneously clustered as one cell would not tend to show path equivalence, as nearby cells tend to have unrelated place fields in spatial tasks (Redish et al., 2001).

\section{Path equivalence, rate remapping, and mixed remapping in CA3}

Although path-equivalent cells fire in similar locations in multiple trajectories, they fire at different peak rates. This pattern of activity is very similar to rate remapping, where individual neurons fire at different rates in different open field environments (Leutgeb et al., 2005b,c, 2006). These two patterns of activity had not been considered to be similar, but we argue that these two types of activity are manifestations of the same basic phenomenon, where individual cells fire in related locations within and across environments.

We found that about half of the cells we recorded fired in only one place or in unrelated locations. In conjunction with the differences in firing rates across locations for path-equivalent cells, our results indicate that each location was still associated with a distinct population pattern of activity. These findings are similar to results from Skaggs et al. (1998), who reported that some, but not all, CA1 place cells had similar place fields across two connected but visually identical open field environments. At the same time, our finding of mixtures of path-equivalent and nonpath-equivalent cells in CA3 stands in contrast to previous re-
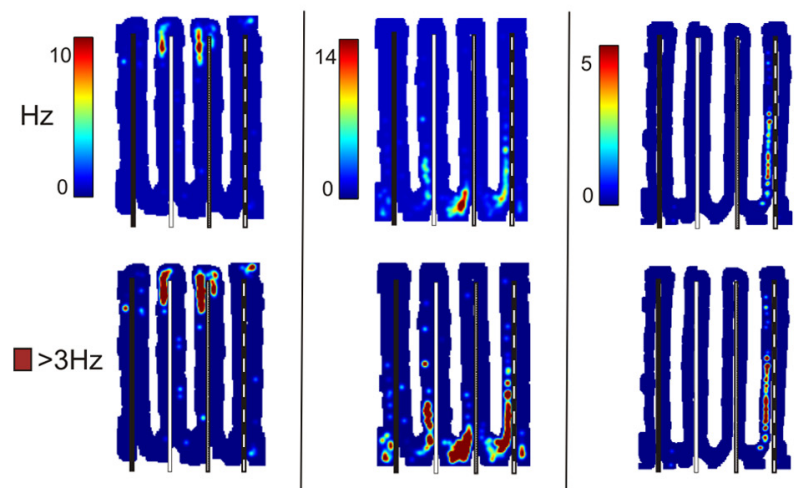

C

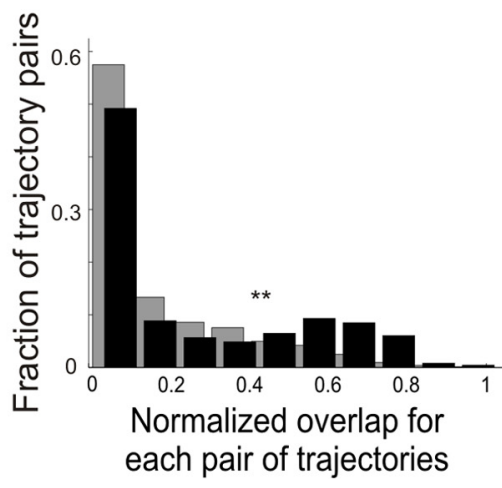

Figure 9. Path-equivalent activity in CA1 neurons active on the multiple-U track. $\boldsymbol{a}, 0$ verhead view of the $U$ track with unique dividers and odors in each U (top left). Spatial firing rate maps of three (A1 cells scaled to the cells' peak firing rate (above) or scaled inearized firing rate of single cells (black) or different cells (light gray) on two different trajectories $\left({ }^{* *} p<0.001\right)$. Only trajectories in the same turn direction were compared.

ports that CA3 activity is frequently coherent: either the majority CA3 cells fire similarly, or the majority fire differently across different locations (Guzowski et al., 2004; Lee et al., 2004; Leutgeb et al., 2004). We therefore suggest that population coherence in $\mathrm{CA} 3$, and the presence of mixed coding properties more generally, will depend on both the specifics of the animal's environment and the task it is performing.

\section{The origin of path-equivalent activity}

Previous work demonstrated that path-equivalent activity in the hippocampus was associated with resets of the EC grid pattern across each repeating geometric element of the environment (Derdikman et al., 2009). Those authors suggested that these resets are sufficient to explain the previous observation of pathequivalent activity in the EC of animals running in the three-arm maze and the presence of path equivalence in the hippocampus. Although it seems likely that grid resets contribute to the presence of path equivalence, this similarity is not sufficient to explain our results. We found that path-equivalent activity in CA3 and CA1 was relatively uncommon in the three-arm maze, despite its previously demonstrated prevalence in the EC (Frank et al., 2000). Thus, path-equivalent activity does not always propagate from the EC to the hippocampus.

Our data suggest instead that both the presence of repeating elements and task-related learning contribute to the presence of path equivalence in the hippocampus. First, our observation of 
path-equivalent activity in the context of the repeating elements of the six-arm maze, and the relative lack of path equivalence in the three-arm maze (which does not contain identical elements), is consistent with the idea that the presence of repeating elements contributes to the development of path-equivalent activity. Second, our finding that path equivalence increases with experience, and in particular that it increases as animals learned the novel sequence 2 in the familiar six-arm maze, demonstrates that path equivalence in the hippocampus depends on experiences relating the paths. Given that the animals had full access to the geometrical structure of the environment for at least five days before their first exposure to S2, the change in path equivalence with experience is more closely aligned to changes in the behavioral significance of individual trajectories than to their stable geometrical character. Therefore, path equivalence increased as animals learned relationships between paths and executed similar behaviors across paths, whether the environment was novel or familiar.

Our results also indicate that path equivalence reflects the development of coordinated neural ensembles. Although neurons in the hippocampus have been shown to fire in the context of coordinated ensembles (Harris et al., 2003; Dragoi and Buzsaki, 2006), the functional role of these ensembles was unknown. We found that hippocampal cells with similar activity across locations are more correlated with one another, suggesting that one role of these ensembles could be to represent related locations. This possibility is consistent with a recent report demonstrating the importance of ensembles for representing multiple reference frames (Keleman and Fenton, 2010). Our analyses indicate that pairs of cells with overlapping, path-equivalent fields across trajectories tended to have high overall short-time-scale correlations of residuals. Similarly, for a given pair of cells with overlapping place fields in one trajectory, more correlated residuals were associated with higher overlaps in another trajectory. So although the similarity of cells' receptive fields in a single location was not predictive of moment-to-moment correlations, similarities over multiple locations were. To our knowledge, this is the first demonstration that the firing properties of two place cells in one location can predict their patterns of spatial activity in another location. We suggest that the fine timescale organization of these cell assemblies can be harnessed to represent learned generalizations across similar locations. As a result, our findings provide a new link between short-time-scale correlations and the representation of specific features of the environment.

\section{The role of path equivalence}

In previous studies, the functional significance of path equivalence and rate remapping was unclear. Our findings support the hypothesis that path equivalence (and thus rate remapping) may be a mechanism to encode common features across related experiences. The development of path equivalence with task learning indicates path-equivalent activity could help the animal link behaviors to specific sets of locations where those behaviors are appropriate (Frank et al., 2000). We suggest that rate remapping across different environments could play the same role in helping the animal associate common features across locations, and thus facilitate learning about any specific relevance of those features. Because previous studies of rate remapping have used only random foraging tasks, a test of that hypothesis will require new studies.

Furthermore, because path-equivalent and non-path-equivalent cells fired together within 100 ms windows, animals could in principle use both types of activity to extract a relative location within each trajectory in the environment as well as a global location from the ensemble of active place cells (Fenton et al., 2008). This would allow hippocampal place cells to simultaneously support memories for unique experiences and learned generalizations across experiences.

\section{References}

Ainge JA, van der Meer MA, Langston RF, Wood ER (2007) Exploring the role of context-dependent hippocampal activity in spatial alternation behavior. Hippocampus 17:988-1002.

Anderson MI, Jeffery KJ (2003) Heterogeneous modulation of place cell firing by changes in context. J Neurosci 23:8827-8835.

Battaglia FP, Sutherland GR, McNaughton BL (2004) Local sensory cues and place cell directionality: additional evidence of prospective coding in the hippocampus. J Neurosci 24:4541-4550.

Cheng S, Frank LM (2008) New experiences enhance coordinated neural activity in the hippocampus. Neuron 57:303-313.

Derdikman D, Whitlock JR, Tsao A, Fyhn M, Hafting T, Moser MB, Moser EI (2009) Fragmentation of grid cell maps in a multicompartment environment. Nat Neurosci 12:1325-1332.

Dragoi G, Buzsaki G (2006) Temporal encoding of place sequences by hippocampal cell assemblies. Neuron 50:145-157.

Fenton AA, Kao HY, Neymotin SA, Olypher A, Vayntrub Y, Lytton WW, Ludvig N (2008) Unmasking the CAl ensemble place code by exposures to small and large environments: more place cells and multiple, irregularly arranged, and expanded place fields in the larger space. J Neurosci 28:11250-11262.

Fox SE, Ranck JBJ (1981) Electrophysiological characteristics of hippocampal complex-spike cells and theta cells. Exp Brain Res 41:399-410.

Frank LM, Brown EN, Wilson MA (2000) Trajectory encoding in the hippocampus and entorhinal cortex. Neuron 27:169-178.

Frank LM, Brown EN, Wilson MA (2001) A comparison of the firing properties of putative excitatory and inhibitory neurons from CA1 and the entorhinal cortex. J Neurophys 86:2029-2040.

Frank LM, Stanley GB, Brown EN (2004) Hippocampal plasticity across multiple days of exposure to novel environments. J Neurosci 24:76817689.

Guzowski JF, Knierim JJ, Moser EI (2004) Ensemble dynamics of hippocampal regions CA3 and CA1. Neuron 44:581-584.

Hampson RE, Simeral JD, Deadwyler SA (1999) Distribution of spatial and nonspatial information in dorsal hippocampus. Nature 402:610-614.

Harris KD, Csicsvari J, Hirase H, Dragoi G, Buzsaki G (2003) Organization of cell assemblies in the hippocampus. Nature 424:552-556.

Hirsh R, Leber B, Gillman K (1978) Fornix fibers and motivational states as controllers of behavior: a study stimulated by the contextual retrieval theory. Behav Biol 22:463-478.

Hsiao S, Isaacson RL (1971) Learning of food and water positions by hippocampus damaged rats. Physiol Behav 6:81-83.

Karlsson MP, Frank LM (2008) Network dynamics underlying the formation of sparse, informative representations in the hippocampus. J Neurosci 28:14271-14281.

Karlsson MP, Frank LM (2009) Awake replay of remote experiences in the hippocampus. Nat Neurosci 12:913-918.

Keleman E, Fenton AA (2010) Dynamic grouping of hippocampal neural activity during cognitive control of two spatial frames. PLoS Biol 8:e1000403.

Kim SM, Frank LM (2009) Hippocampal lesions impair rapid learning of a continuous spatial alternation task. PLoS One 4:e5494.

Lee D, Port NL, Kruse W, Georgopoulos AP (1998) Variability and correlated noise in the discharge of neurons in motor and parietal areas of the primate cortex. J Neurosci 18:1161-1170.

Lee I, Yoganarasimha D, Rao G, Knierim JJ (2004) Comparison of population coherence of place cells in hippocampal subfields CA1 and CA3. Nature 430:456-459.

Leutgeb JK, Leutgeb S, Treves A, Meyer R, Barnes CA, McNaughton BL, Moser MB, Moser EI (2005a) Progressive transformation of hippocampal neuronal representations in "morphed" environments. Neuron 48:345-358.

Leutgeb S, Leutgeb JK, Treves A, Moser MB, Moser EI (2004) Distinct ensemble codes in hippocampal areas CA3 and CA1. Science 305:1295-1298.

Leutgeb S, Leutgeb JK, Barnes CA, Moser EI, McNaughton BL, Moser MB 
(2005b) Independent codes for spatial and episodic memory in hippocampal neuronal ensembles. Science 309:619-623.

Leutgeb S, Leutgeb JK, Moser MB, Moser EI (2005c) Place cells, spatial maps and the population code for memory. Curr Opin Neurobiol 15:738-746.

Leutgeb S, Leutgeb JK, Moser EI, Moser MB (2006) Fast rate coding in hippocampal CA3 cell ensembles. Hippocampus 16:765-774.

Lever C, Wills T, Cacucci F, Burgess N, O’Keefe J (2002) Long-term plasticity in hippocampal place-cell representation of environmental geometry. Nature 416:90-94.

McHugh TJ, Jones MW, Quinn JJ, Balthasar N, Coppari R, Elmquist JK, Lowell BB, Fanselow MS, Wilson MA, Tonegawa S (2007) Dentate gyrus NMDA receptors mediate rapid pattern separation in the hippocampal network. Science 317:94-99.

Morris RG, Garrud P, Rawlins JN, O’Keefe J (1982) Place navigation impaired in rats with hippocampal lesions. Nature 297:681-683.

Muller RU, Kubie JL (1987) The effects of changes in the environment on the spatial firing of hippocampal complex-spike cells. J Neurosci 7:1951-1968.

Nakazawa K, Quirk MC, Chitwood RA, Watanabe M, Yeckel MF, Sun LD, Kato A, Carr CA, Johnston D, Wilson MA, Tonegawa S (2002) Requirement for hippocampal CA3 NMDA receptors in associative memory recall. Science 297:211-218.

O'Keefe J, Nadel L (1978) The hippocampus as a cognitive map. London: Oxford UP.

Packard MG, McGaugh JL (1996) Inactivation of hippocampus or caudate nucleus with lidocaine differentially affects expression of place and response learning. Neurobiol Learn Mem 65:65-72.

Quirk MC, Wilson MA (1999) Interaction between spike waveform classification and temporal sequence detection. J Neurosci Methods 94:41-52.

Redish AD, Battaglia FP, Chawla MK, Ekstrom AD, Gerrard JL, Lipa P,
Rosenzweig ES, Worley PF, Guzowski JF, McNaughton BL, Barnes CA (2001) Independence of firing correlates of anatomically proximate hippocampal pyramidal cells. J Neurosci 21:RC134.

Schoppik D, Nagel KI, Lisberger SG (2008) Cortical mechanisms of smooth eye movements revealed by dynamic covariations of neural and behavioral responses. Neuron 58:248-260.

Shadlen MN, Newsome WT (1998) The variable discharge of cortical neurons: implications for connectivity, computation, and information coding. J Neurosci 18:3870-3896.

Singer AC, Frank LM (2009) Rewarded outcomes enhance reactivation of experience in the hippocampus. Neuron 64:910-921.

Skaggs WE, McNaughton BL (1998) Spatial firing properties of hippocampal CA1 populations in an environment containing two visually identical regions. J Neurosci 18:8455-8466.

Skaggs WE, McNaughton BL, Gothard K, Markus EJ (1993) An information-theoretic approach to deciphering the hippocampal code. In: Advances in neural information processing 5 (Hanson SJ, Cowan JD, Giles CL, eds), pp 1030-1037. San Mateo, CA: Morgan Kaufman.

Smith AC, Frank LM, Wirth S, Yanike M, Hu D, Kubota Y, Graybiel AM, Suzuki WA, Brown EN (2004) Dynamic analysis of learning in behavioral experiments. J Neurosci 24:447-461.

Smith AC, Wirth S, Suzuki WA, Brown EN (2007) Bayesian analysis of interleaved learning and response bias in behavioral experiments. J Neurophysiol 97:2516-2524.

Tanila H (1999) Hippocampal place cells can develop distinct representations of two visually identical environments. Hippocampus 9:235-246.

Wills TJ, Lever C, Cacucci F, Burgess N, O’Keefe J (2005) Attractor dynamics in the hippocampal representation of the local environment. Science 308:873-876. 